\title{
Convergent Perturbation Expansions for Euclidean Quantum Field Theory
}

\author{
Gerhard Mack and Andreas Pordt
}

II. Institut für Theoretische Physik der Universität Hamburg, D-2000 Hamburg 50, Federal Republic of Germany

\section{Dedicated to the memory of Kurt Symanzik}

\begin{abstract}
Mayer perturbation theory is designed to provide computable convergent expansions which permit calculation of Greens functions in Euclidean quantum field theory to arbitrary accuracy, including "nonperturbative" contributions from large field fluctuations. Here we describe the expansions at the example of 3-dimensional $\lambda \phi^{4}$-theory (in continuous space). They are not essentially more complicated than standard perturbation theory. The $n^{\text {th }}$ order term is expressed in terms of $O(n)$-dimensional integrals, and is of order $\lambda^{k}$ if $4 k-3 \leqq n \leqq 4 k$.
\end{abstract}

\section{Introduction}

Mayer perturbation theory is designed to provide computable convergent expansions which permit calculation of Greens functions in Euclidean quantum field theory to arbitrary accuracy, including "nonperturbative contributions" from large field fluctuations. Their $n^{\text {th }}$ order term is given by $O(n)$-dimensional integrals, as is the case in standard perturbation theory. In principle such expansions have a chance of converging for asymptotically free theories - including superrenormalizable ones - for problems where a small coupling constant is effective. Some models will require a more sophisticated treatment of the large field region, though. In this paper we describe the expansions in their simplest form at the example of weakly coupled massive $\lambda \phi^{4}$-theory in $v=3$ dimensions. Part I of the paper presents the main ideas and constructions. It describes the expansions in an elementary way, and discusses the relation to standard perturbation theory: The sum of all terms in the Mayer expansion up to order $4 n$ equals the sum of all renormalized Feynman diagrams up to order $n$, plus a computable correction of higher order or nonperturbative origin. The raison d'être of Mayer expansions is their computability and convergence. Here we concentrate on computability. We plan to present estimates and discuss convergence properties in a subsequent article (Part II).

Mayer expansions [i.e., iterative solutions of Kirkwood Salsburg or Mayer Montroll equations, in place of Schwinger Dyson equations] were introduced into 
quantum field theory in Symanzik's celebrated paper on Euclidean quantum field theory [1]. Brydges and Federbush [2] used Mayer expansions to obtain an effective action for a Coulomb gas. The Mayer expansions on the staggered lattice that we are going to present here are equivalent to iterated Mayer expansions using successively lower lattice cutoffs (cf. end of Sect.2). Iterated Mayer expansions with Pauli-Villars cutoff were developped by Göpfert and Mack in $[3,4]$, and applied to prove permanent confinement of static quarks in 3-dimensional U(1) lattice gauge theory. The work presented here is a continuation of the program started there, but the paper will be selfcontained. The expansions presented here are examples of what is called a phase cell cluster expansion in constructive field theory. Phase cell cluster expansions were introduced by Glimm and Jaffe [5] and further developped by Magnen and Sénéor [6] and Battle and Federbush [7, 8]. Our convergence proof (in preparation) combines elements of the analysis of Magnen and Sénéor, and of [4], with a UV-stability argument (the mass counterterm is dominated by the quartic interaction, cf. below). Elements of the exact renormalization group approach of Kupiainen and Gawedzki [9] are also incorporated ${ }^{1}$.

The space on which the theory lives originally [continuum $\mathbb{R}^{v}$ or cubic lattice $(a \mathbb{Z})^{v}$ of lattice spacing $\left.a\right]$ will be called the base space. The generating function $\ln Z(\psi)$ of the connected free-propagator-amputated Euclidean Greens functions depends on a field $\psi$ on base space. Its derivatives at $\psi=0$ give the Greens functions, see Appendix A. In Mayer perturbation theory they are computed from an expansion of the form

$$
\ln Z(\psi)=\sum_{n \geqq 0} \int_{x_{1}} \ldots \int_{x_{n} \in \Lambda} \tilde{\mathscr{M}}\left(x_{1}, \ldots, x_{n} \mid \psi\right) .
$$

The resulting expansions for Greens functions become particularly simple when suitable normalization conditions are imposed, see Eqs. (1.16a), (1.19) below. The leading term $n=0$ is given by

$$
\tilde{\mathscr{M}}(\emptyset \mid \psi)=-\frac{\lambda}{4 !} \int_{\text {base }} \psi(z)^{4} .
$$

The integrations over $x_{i}$ in the later terms are actually summations over all points $x$ of a staggered lattice $\Lambda$ that is superimposed on the base space (see Fig. 1 ). The staggered lattice $\Lambda=\left(\Lambda_{0}, \Lambda_{1}, \Lambda_{2}, \ldots\right)$ consists of a finite or infinite sequence [disjoint union] of lattices $\Lambda_{j}$ of decreasing lattice spacing $a_{j}$. For instance, $a_{j}=L^{-j} a_{0}$, where $L$ is a suitably chosen integer $>1$ and $a_{0}$ is the physical length scale $^{2}$. A point $x \in \Lambda$ is an element of $\Lambda_{j}$ for some $j \geqq 0$. In the presence of a lattice cutoff $a$, there is a finest lattice $\Lambda_{N}$ with lattice spacing $a_{N}=a$. For a continuum theory $N=\infty$ - i.e. the sequence is infinite - and the staggered lattice admits as maps a semigroup of scale transformations by integer powers of $L^{-1}$ in addition to

1 In recent announcements, Feldman, Magnen, Rivasseau, and Sénéor promise an improved version of the expansions of [6], with application to the infrared behavior of massless lattice $\lambda \phi^{4}$-theory in 4 dimensions [10], and Kupiainen and Gawedzki describe how they handle the same model [11]. This supports the belief that asymptotically free renormalizable models are not necessarily intrinsically more difficult than superrenormalizable ones

2 We believe that $L=2$ is an optimal choice yet 


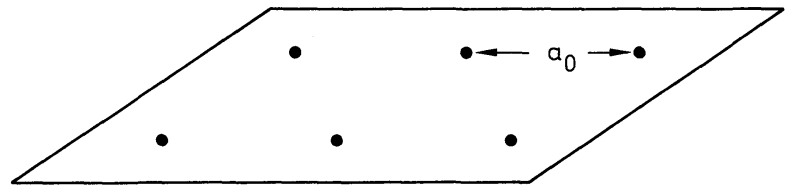

$\Lambda_{0}$

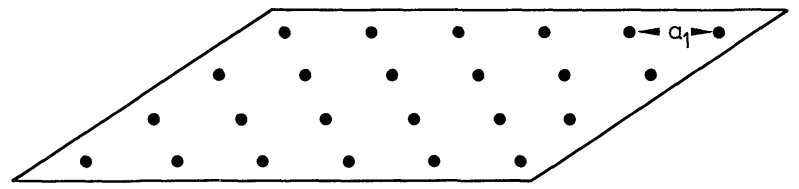

$\Lambda_{1}$

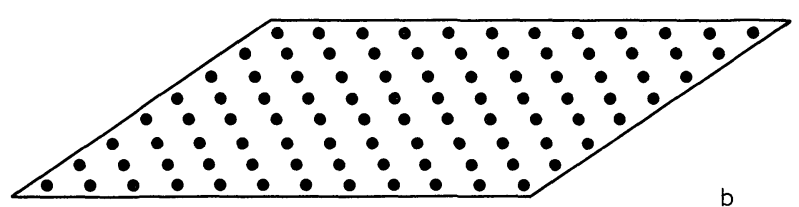

$\wedge_{2}$

b

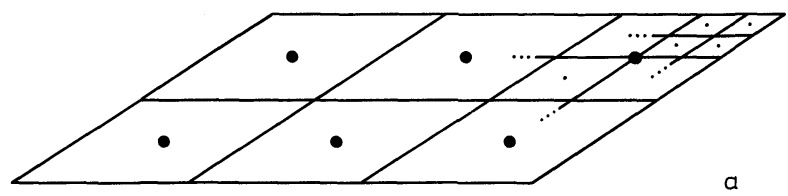

base

Fig. 1a and b. The staggered lattice. A point $x \in \Lambda_{j}$ of the staggered lattice may be regarded as a cube of side length $a_{j}$ in base space. The cubes are positioned in such a way that smaller cubes fit into larger ones (i.e. do not intersect their boundaries). a base space with lattices of side length $a_{j}=L^{-j} a_{0}$ superimposed, $j=0,1,2, \ldots . \mathbf{b}$ Every cube in one of the lattices that is superimposed on base space is represented by a dot in this pictorial representation of the staggered lattice $\Lambda=\Lambda_{0}+\Lambda_{1}+\Lambda_{2}+\ldots$. The dots in layer $\Lambda_{j}$ represent cubes of side length $a_{j}$. (Drawing for 2 dimensions)

the group of translations by integer multiples of $a_{0}$. Integration over the staggered lattice includes a summation over length scales $j$. In $v$ dimensions

$$
\int_{x \in \Lambda}(\ldots)=\sum_{j=0}^{N} a_{j}^{v} \sum_{x \in \Lambda_{j}}(\ldots) .
$$

The augmented Mayer amplitudes $\tilde{\mathscr{M}}\left(x_{1}, \ldots, x_{n} \mid \psi\right)$ are finite sums of products of Mayer amplitudes $\mathscr{M}\left(x_{i_{1}}, \ldots, x_{i_{k}} \mid \psi\right)$ with $k \leqq n$, multiplied with combinatorial coefficients. The general formula is given in Eqs. (3.6) of Sect. 3. The Mayer amplitudes $\mathscr{M}$ are defined for finite subsets $X=\left\{x_{1}, \ldots, x_{n}\right\} \subset \Lambda$ of the staggered lattice. That is, they are symmetrical in their arguments and defined only when all their arguments are distinct. They are defined by considering auxiliary theories in which the interaction has been switched off, in a suitable way, outside finite subsets $X$ of the staggered lattice. For 3-dimensional $\lambda \phi^{4}$-theory this is done as follows. 
The interaction of the model is $V(\phi)-\delta V(\nu \mid \phi)$,

$$
\begin{gathered}
V(\phi)=\frac{\lambda}{4 !} \int_{\text {base }} \phi(z)^{4}, \\
\delta V(v \mid \phi)=\delta e(v)+\int_{\text {base }} \frac{1}{2} \delta m^{2}(v \mid z) \phi(z)^{2} .
\end{gathered}
$$

It is expedient to consider the counterterms as functions of the free propagator $v$ of the theory. In our model [and, more generally, in asymptotically free theories], the counterterms are known a priori. For instance

$$
\delta m^{2}(v \mid z)=\frac{1}{2} \lambda v(z, z)-\frac{1}{3 !} \lambda^{2} \int_{z^{\prime} \in \text { base }} v\left(z, z^{\prime}\right)^{3} .
$$

There is a similar expression for the field independent ("vacuum energy") counterterm $\delta e$. Alternatively, $\delta e$ may be fixed by imposing normalization conditions. One may also speed up UV-convergence by making oversubstractions, i.e. include wave function and coupling constant renormalization counterterms, and higher order terms in (1.4), if one wishes to. When the propagator $v$ is translation invariant, then $\delta m^{2}$ is independent of $z$. Both counterterms vanish when $v=0$.

One splits the field $\phi$ into pieces $\varphi^{j}$ that are constant on cubes of side length $a_{j}$. The points $x \in \Lambda_{j}$ of the staggered lattice may be identified with cubes in the base space of side length $a_{j}$. As a result, the pieces $\varphi^{j}$ specify a field on the staggered lattice, viz. $\varphi(x)=\varphi^{j}(z)$ for $z \in x, x \in \Lambda_{j}$. The decomposition reads

$$
\phi(z)=\int_{x \in \Lambda} \mathscr{A}(z, x) \varphi(x) \equiv \mathscr{A} \varphi(z) \text { for } z \in \text { base. }
$$

The kernels $\mathscr{A}$ are determined by a choice of block spin (and the free propagator $v$ ). To be specific, let us choose the block spin on the lattice $\Lambda_{j}$ of lattice spacing $a_{j}$ as

$$
\Phi^{j}(x)=\underset{z \in x}{a v} \phi(z) \equiv a_{j}^{-v} \int_{z \in x} \phi(z) \text { for } x \in \Lambda_{j} .
$$

Then the kernels $\mathscr{A}$ are the same as in the work of Kupiainen and Gawedzki (except for the presence of a mass) [9], see Appendix B. There is a corresponding split of the free propagator

$$
v\left(z_{1}, z_{2}\right)=\iint_{x_{1}, x_{2} \in \Lambda} \mathscr{A}\left(z_{1}, x_{1}\right) v\left(x_{1}, x_{2}\right) \mathscr{A}\left(z_{2}, x_{2}\right) \text { for } z_{1}, z_{2} \in \text { base. }
$$

The propagator $v\left(x_{1}, x_{2}\right)$ on the staggered lattice vanishes unless $x_{1}$ and $x_{2}$ are in the same layer. Both $\mathscr{A}(z, x)$ and $v\left(x_{1}, x_{2}\right)$ decay exponentially with distance [between $z$ and $x \subset$ base, or between $x_{1}$ and $x_{2}$ ] with decay length ${ }^{3} a_{j}$ if $x \in \Lambda_{j}$, $x_{1,2} \in \Lambda_{j+1}$, see [9]. The decay of the propagator on $\Lambda_{0}$ is determined by the mass. The propagator $v$ is positive semi-definite.

The interaction may be considered as a function of the field $\varphi$ on the staggered lattice. We set

$$
\begin{aligned}
\mathfrak{B}(\psi, \varphi) & =V(\psi+\phi)-\frac{\lambda}{4 !} \int_{\text {base }} \psi(z)^{4}, \\
\delta \mathfrak{B}(\vartheta \mid \psi, \varphi) & =\delta V(v \mid \psi+\phi),
\end{aligned}
$$

3 This implies that the propagator of $\varphi^{j}$ has infrared cutoff $a_{j-1}^{-1}$, while a UV-cutoff $a_{j}^{-1}$ is supplied by the lattice $\Lambda_{j}$ 
when $\phi$ and $\varphi$ are related by Eq. (1.5). [The external field $\psi$ on base space is argument of the generating function for amputated Greens functions.]

While it is custumary to call $V$ the interaction, the reader is advised to think of interaction as being mediated by propagators. The interaction is switched off by putting the propagator equal to zero outside $X$. Given a subset $X$ of the staggered lattice, let $\chi_{X}$ be its characteristic function

$$
\chi_{X}(x)= \begin{cases}1 & \text { for } x \in X \\ 0 & \text { otherwise }\end{cases}
$$

and define $X$-dependent propagators by

$$
\begin{aligned}
& v_{X}\left(x_{1}, x_{2}\right)=\chi_{X}\left(x_{1}\right) v\left(x_{1}, x_{2}\right) \chi_{X}\left(x_{2}\right) \\
& v_{X}\left(z_{1}, z_{2}\right)=\iint_{x_{1}, x_{2} \in A} \mathscr{A}\left(z_{1}, x_{1}\right) v_{X}\left(x_{1}, x_{2}\right) \mathscr{A}\left(z_{2}, x_{2}\right) .
\end{aligned}
$$

Partition functions $Z_{1}(X \mid \psi)$ for finite subsets $X \subset \Lambda$ are defined using as free field measure the Gaussian measure $d \mu_{v_{X}}(\varphi)$ with propagator [covariance] $v_{X}$. Because the propagator vanishes outside $X$ this measure is supported on fields $\varphi=\chi_{X} \varphi$ that vanish outside $X$. Therefore the integral is effectively $n$-dimensional when $X$ contains $n$ points [cf. Eq. (3.15) of Sect. 3]. The interaction $\mathfrak{B}$ is as before, but the counterterms are adjusted by substituting $v_{X}$ for $v$ in the argument of $\delta \mathfrak{B}$.

$$
Z_{1}(X \mid \psi)=\int d \mu_{v_{X}}(\varphi) \exp \left[-\mathfrak{B}(\psi, \varphi)+\delta \mathfrak{B}\left(v_{X} \mid \psi, \varphi\right)\right]
$$

Our definitions are such that

$$
Z_{1}(\emptyset \mid \psi)=1
$$

The generating function for the amputated Green functions is obtained as an " $\infty$-volume limit" $X \nearrow \Lambda$ (see end of Appendix B)

$$
\ln Z(\psi)=-\frac{\lambda}{4 !} \int_{\text {base }} \psi(z)^{4}+\ln Z_{1}(\Lambda \mid \psi) \quad(+ \text { const })
$$

This switchoff of interaction and induced counterterms on part of the staggered lattice is the central idea of this paper. It is the key to computability and simplicity. The adjustment of the counterterms becomes natural when the interaction is viewed as mediated by propagators. It is also essential in order to maintain stability. In and close to the continuum the bare mass squared is negative. The mass counterterm must therefore be dominated by the quartic interaction. The basic inequality is

$$
\sup _{\phi} \int_{\text {base }}\left[-\frac{\lambda}{4 !} \phi(z)^{4}+\frac{1}{2} \delta m^{2}(z) \phi(z)^{2}\right] \leqq 6 \lambda^{-1} \int_{\text {base }}\left[\delta m^{2}(z)\right]^{2} .
$$

When the counterterm is switched off outside $X, \lambda^{-1} \int\left[\delta m^{2}\right]^{2}$ becomes of the order of $\lambda$. "volume of $X$ " and its exponential can be controlled by powers of $\lambda$ in suitable units.

Let us return to the computation of Mayer amplitudes $\mathscr{M}$. They are defined by requiring validity of the following relations for arbitrary finite nonempty subsets $X$ 
of the staggered lattice $\Lambda$. Set

$$
A(X \mid \psi)=n ! a\left(x_{1}\right)^{v} \ldots a\left(x_{n}\right)^{v} \mathscr{M}\left(x_{1}, \ldots, x_{n} \mid \psi\right)+\delta_{1, n}
$$

for

$$
X=\left\{x_{1}, \ldots, x_{n}\right\}, \quad \text { with } a(x) \equiv a_{j} \text { for } x \in \Lambda_{j} .
$$

The dimension $v=3$ in our model. We demand

$$
Z_{1}(X \mid \psi)=\sum_{X=\Sigma Y} \prod_{Y} A(Y \mid \psi) \text { for all finite } X .
$$

Summation is over all partitions of $X$ into disjoint nonempty subsets $Y$. Equations (1.9b), (1.12b) exhibit $Z_{1}(X)$ as partition function of a polymer system with activities $A(Y)$ (see Sect. 2). It is well known that Eq. (1.12b) has a converse which determines the activity $A(Y)$ uniquely, given the partition functions $Z_{1}(X)$ for $X \subseteq Y$.

$$
A(Y \mid \psi)=\sum_{n \geqq 1}(-1)^{n-1}(n-1) ! \sum_{Y=\Sigma_{1}^{n} X_{i}} \prod_{i} Z_{1}\left(X_{i} \mid \psi\right) .
$$

For practical calculations (and for deriving estimates) one uses other more convenient formulae for the Mayer amplitudes in place of Eq. (1.12c). They will be described in the following sections. It is now clear, however, that the Mayer amplitudes $\mathscr{M}\left(x_{1}, \ldots, x_{n} \mid \psi\right)$ are computable in terms of $\leqq n$-dimensional integrals, because expression (1.9a) is an integral over $n$ real variables $\varphi\left(x_{i}\right)$ when $X=\left\{x_{i}\right\}$ has $n$ points.

Let us note, for comparison, that standard perturbation theory can also be put on a staggered lattice. This can be done Feynman graph by Feynman graph. Consider for instance a $n^{\text {th }}$ order graph with 4-leg vertices only. The Feynman integrand in coordinate space is a product of propagators $v\left(z_{i}, z_{j}\right)$, multiplied with $(-\lambda)^{n}$ and possibly with a combinatorial factor. Split the propagator as in Eq. (1.6) and imagine that the $z_{j}$-integration over base space are done. This produces nonlocal vertex functions on the staggered lattice,

$$
\mathscr{V}_{4}\left(x_{1}, \ldots, x_{4}\right)=-\frac{\lambda}{4 !} \int_{z \in \text { base }} \mathscr{A}\left(z, x_{1}\right) \ldots \mathscr{A}\left(z, x_{4}\right), \quad \text { etc. }
$$

The Feynman integral becomes an integral over $4 n$ arguments $x_{i}$ in the staggered lattice. It can be rewritten as a sum of "integrals" over $k \leqq n$ arguments $y_{i}$ that assume distinct values in $\Lambda$ (cf. Sect. 4).

Now we will explain, briefly, how our renormalization procedure with $X$-dependent counterterms works. We begin with a perturbation theoretic illustration. For simplicity we will ignore tadpoles and the $O(\lambda)$ piece of the mass counterterm that cancels them. To order $\lambda^{2}$ the generating function $\ln Z(\psi)$ is a polynomial in $\psi$, and its quadratic term in $\psi$ is given by

$$
\ln Z(\psi)=\iint_{z_{1}, z_{2} \in \text { base }} \psi \ldots \bigodot_{z_{1}} \ldots \psi-\int_{z_{2} \in \text { base }} \psi \ldots \underset{z_{1}}{\times} \ldots \psi+\ldots
$$

in graphical notation, with

$$
\underset{z_{1}}{\times}=\int_{z_{2} \in \text { base }} \psi \bigcap_{z_{1}}^{z_{2}} \ldots \psi .
$$


Inserting the expression for the counterterm gives

$$
\begin{aligned}
\ln z(\psi) & =\iint_{z_{1}, z_{2} \in \text { base }}\left[\psi \ldots \bigodot_{z_{1}} \ldots \psi-\psi \ldots \bigwedge_{z_{2}}^{z_{2}} \ldots \psi\right] \\
& =\frac{(-\lambda)^{2}}{3 !} \iint_{z_{1}, z_{2}} \psi\left(z_{1}\right)\left[\psi\left(z_{2}\right)-\psi\left(z_{1}\right)\right] v\left(z_{1}, z_{2}\right)^{3} .
\end{aligned}
$$

The counterterm differs from the main term by reversal of sign and a "shift of the field" $\psi$ from $z_{2}$ to $z_{1}$. Such "shift of the field" is also the basic operation in the recent work of Gallavotti and Nicoló [12] on perturbation theoretic renormalization. In coordinate space language, the logarithmic UV-divergence of the self mass in continuous space appears in the guise of an ill defined product of distributions $v\left(z_{1}, z_{2}\right)^{3}$ which contains a divergent piece proportional $\delta\left(z_{1}-z_{2}\right)$ [13]. Expression (1.15) is a well defined distribution, however, for smooth fields $\psi$, because of the zero of the factor $\left[\psi\left(z_{2}\right)-\psi\left(z_{1}\right)\right]$ at $z_{2}=z_{1}$.

To put the renormalized Feynman integral (1.15a) on the staggered lattice, we perform the split (1.6) of the propagator $v$ in the factor $v\left(z_{1}, z_{2}\right)^{3}$ in expression (1.15b). This amounts to splitting the main term and the counterterm simultaneously, and combining the two terms piecewise in the obvious fashion. The split introduces integrations over six variables $x_{1}^{a}, x_{2}^{a}(a=1,2,3)$ on the staggered lattice, and we imagine that the two integrations of $z_{1}, z_{2}$ over the base space are done first. [They converge because restricted propagators $v_{X}\left(z_{1}, z_{2}\right)$ are less singular at $z_{1}, z_{2}$ than $v\left(z_{1}, z_{2}\right)$, by positivity.] After the split, UV-divergences would show up as divergences in the sums over scales $j$ as $j \rightarrow \infty$. Such sums are implied in the integrations (1.2) over the staggered lattice. Consider now a term in the integrals (actually sums) over $\Lambda$ which involves a propagator $v\left(x_{1}, x_{2}\right)$ with $x_{1}$, $x_{2} \in \Lambda_{j}$. It appears in the combination $\mathscr{A}\left(z_{1}, x_{1}\right) v\left(x_{1}, x_{2}\right) \mathscr{A}\left(z_{2}, x_{2}\right) \equiv \hat{v}\left(z_{1}, z_{2}\right)$. Because of the decay properties of kernels $\mathscr{A}$ and $v$, the restricted propagator $\hat{v}$ becomes exponentially small with distance between $z_{1}$ and $z_{2}$ with decay length $a_{j}$. Suppose now that the field $\psi$ is smooth on length scale $a_{k}$ for some $k<j$. The zero of the factor $\left[\psi\left(z_{2}\right)-\psi\left(z_{1}\right)\right]$ in expression (1.15b) will then give rise to a suppression factor $a_{j} / a_{k}$. This suppression factor is enough to eliminate the logarithmic divergence in the sum over length scales $j \rightarrow \infty$ that would be present if the mass counterterm had not been included in (1.15).

Self mass graphs (1.15a) may also appear as subgraphs of larger graphs. In this case the factor $\psi\left(z_{1}\right) \psi\left(z_{2}\right)$ is replaced by some function $f\left(z_{1}, z_{2}\right)$ that is determined by the rest of the graph. Since potential UV-divergences would show up as divergences in the sum over length scales $a_{j}$ as $j \rightarrow \infty$ (where $a_{j}$ is the decay rate of the fastest decaying restricted propagator in the subgraph) one needs only consider the case that $f\left(z_{1}, z_{2}\right)$ is smooth on some length scale $a_{k}$, with $k<j$. ( $k$ is determined by the restricted propagators that appear in the rest of the graph. $)^{4}$ The effect of the counterterm is to substitute $f\left(z_{1}, z_{2}\right)-f\left(z_{1}, z_{1}\right)$ for $f\left(z_{1}, z_{2}\right)$. This gives rise to a convergence producing suppression factor $a_{j} / a_{k}$ as before.

4 In the perturbative renormalization theory of Gallavotti and Nicoló [12] ordering of lines by frequency $j$ replaces the Zimmermann forests [13] 
Our discussion of renormalization has been perturbative so far. Our Mayer amplitudes may be thought of as given by low order Feynman graphs (split as described above) plus corrections. The corrections turn out not to produce new UV-divergences because they have explicit extra factors $a_{j}$. In conclusion, renormalization suppresses dangerous contributions to individual Mayer amplitudes $\mathscr{M}\left(x_{1}, \ldots, x_{n} \mid \psi\right)$ with some arguments $x_{r}$ in very fine lattices $\Lambda_{j}(j$ large). This works in such a way that the sums over length scales that have to be done as part of the integrations in (1.1) become convergent.

In the analysis of Magnen and Sénéor [6], part of the UV-convergence factors $L^{-j}$ is used up to control combinatorial factors. They arise because a large cube $x^{\prime} \in \Lambda_{k}$ may be coupled to many smaller cubes $x, x^{\prime} \supset x \in \Lambda_{j}, j>k$.

We conclude our introductory discussion of renormalization with a remark about renormalization conditions. The $X$-dependent mass- and vacuum energy counterterms $\delta m^{2}$ and $\delta e$ are chosen in such a way that the following renormalization conditions are approximately fulfilled, for all finite nonempty subsets $X=\left\{x_{1}, \ldots, x_{n}\right\}$ of $\Lambda$ and all $z \in$ base.

$$
\begin{gathered}
\mathscr{M}\left(x_{1}, \ldots, x_{n} \mid \psi=0\right)=0 \\
\int_{z^{\prime} \in \text { base }} \frac{\delta^{2}}{\delta \psi\left(z^{\prime}\right) \delta \psi(z)} \mathscr{M}\left(x_{1}, \ldots, x_{n} \mid \psi=0\right)=0 .
\end{gathered}
$$

The renormalization conditions (1.16) are not "too many" because there are counterterms $\delta e(X)$ and $\delta m^{2}(X \mid z)$ to be fixed for arbitrary $X \subset \Lambda$ and $z \in$ base.

Exact validity of Eq. (1.16a) may be enforced by fixing the constant term $\delta e(X)$ in the action such that

$$
Z_{1}(X \mid \psi=0)=1 \text { for all } X \text {. }
$$

If this is done, then the expansions for the Greens functions become particularly simple. The full, free-propagator-amputated Greens functions (including disconnected parts) are given by

$$
\begin{aligned}
G\left(\underline{z}_{1}, \ldots, \underline{z}_{k}\right) & =Z(\Lambda \mid \psi=0)^{-1} \frac{\delta^{k}}{\delta \psi\left(z_{1}\right) \ldots \delta \psi\left(z_{k}\right)} Z(\Lambda \mid \psi=0), \\
Z(X \mid \psi) & \equiv Z_{1}(X \mid \psi) \exp \left[-\frac{\lambda}{4 !} \int_{\text {base }} \psi(z)^{4}\right]
\end{aligned}
$$

Performing the differentations in expression (1.12b) one obtains with Eqs. (1.12a), (1.16a), and (1.17),

$$
\begin{aligned}
G\left(\underline{z}_{1}, \underline{z}_{2}\right)= & \sum_{n \geqq 1} \int_{x_{1} \ldots x_{n} \in \Lambda}\left[\frac{\delta^{2}}{\delta \psi\left(z_{1}\right) \delta \psi\left(z_{2}\right)} \mathscr{M}\left(x_{1}, \ldots, x_{n} \mid \psi=0\right)\right. \\
& \left.+\sum_{k=1}^{n-1}\left(\begin{array}{l}
n \\
k
\end{array}\right) \frac{\delta}{\delta \psi\left(z_{1}\right)} \mathscr{M}\left(x_{1}, \ldots, x_{k} \mid \psi=0\right) \frac{\delta}{\delta \psi\left(z_{2}\right)} \mathscr{M}\left(x_{k+1}, \ldots, x_{n} \mid \psi=0\right)\right]
\end{aligned}
$$

and similar formulae for the higher $n$-point functions. In Eq. (1.19) the second term in [] is actually zero by symmetry. 
The free-propagator-amputated Greens functions $G\left(\underline{x}_{1}, \ldots, \underline{x}_{n}\right)$ are not 1-particle irreducible, and neither are the Mayer amplitudes $\mathscr{M}$. 1-particle irreducible Mayer amplitudes $\mathscr{M}^{(1)}$ can be introduced by the following defining relation. We write it for activities $A$ which are related to Mayer amplitudes $\mathscr{M}$ by Eq. (1.12a).

$$
\begin{aligned}
A(X \mid \psi)= & A^{(1 i)}(X \mid \psi) \\
& +\sum_{X=X_{1}+X_{2}} \int_{z_{1}, z_{2} \in \text { base }} \int_{\substack{x_{1} \in X_{1} \\
x_{2} \in X_{2}}} \frac{\delta A^{(1 i)}\left(X_{1} \mid \psi\right)}{\delta \psi\left(z_{1}\right)} \\
& \cdot \mathscr{A}\left(z_{1}, x_{1}\right) v\left(x_{1}, x_{2}\right) \mathscr{A}\left(z_{2}, x_{2}\right) \frac{\delta A\left(X_{2} \mid \psi\right)}{\delta \psi\left(z_{2}\right)} .
\end{aligned}
$$

Summation is over partitions of $X$ in two nonempty subsets $X_{1}$ and $X_{2}$. Renormalization conditions (1.16) carry over to the one particle irreducible amplitudes $\mathscr{M}^{(1)}$ (and also to $\tilde{\mathscr{M}}$ ).

One may want to impose also the second renormalization condition (1.16b) exactly. Description of such an alternative renormalization scheme is outside the scope of the present paper.

Our method may also be used to compute effective actions in the sense of Wilson [15], and corresponding Boltzmann factors, for lattice cutoffs $a_{j}$. They depend on a block spin $\Phi^{j}$ on $\Lambda_{j}$. Set

$$
\Lambda_{[>j]}=\sum_{k>j} \Lambda_{j} \quad \text { and } \quad \Phi^{j}(x)=0 \text { for } x \notin \Lambda_{j} .
$$

Then $\ln Z\left(\Lambda_{[>j]} \mid \mathscr{A} \Phi^{j}\right)$ and $Z\left(\Lambda_{[>j]} \mid \mathscr{A} \Phi^{j}\right)$, which are determined by Mayer amplitudes $\mathscr{M}(X \mid \psi)$ with $X \subset \Lambda_{[>j]}$, are almost the desired quantities. ["Almost" because one needs to correct for the fact that the "partial bare mass" $m_{0}^{2}(z)=m^{2}$ $-\delta m^{2}\left(v_{\Lambda[>j]} \mid z\right)$ need not be exactly invariant under continuous translations.] This is of interest for theories which are not weakly coupled at the physical length scale $a_{0}$. We hope to come back to this problem in a future paper. Ultimately one may hope to design a Monte Carlo procedure for continuum theories in which correction terms in the effective Boltzmann factor are picked randomly.

The plan of the rest of the paper is as follows: In Sect. 2 we recall results of the theory of polymer systems, and formulae for and properties of truncated expectation values. In Sect. 3 we show how the Mayer amplitudes can be computed as truncated expectation values of so-called "molecular activities" $B$ that are determined by the interaction $V$ and the kernels $\mathscr{A}$ (i.e. propagator $v$ and block spin). Formulae for and properties of these molecular activities are discussed. The result is summarized in Theorem 3.3. Section 4 discusses the relation with perturbation theory. It includes a pedagogical discussion of the relation between standard perturbation theory and Mayer expansions on a simple (as opposed to staggered) lattice. In Sect. 5 the trivial, but still instructive, model of $\lambda \phi^{4}$-theory on a lattice with only one point is considered.

\section{Theory of Polymer Systems}

Following Gruber and Kunz [16], a polymer system on a finite or countable set $\Lambda$ is specified as follows. The elements of $\Lambda$ are called sites. Certain finite nonempty 
subsets $P$ of $\Lambda$ are declared to be polymers. Among them are the monomers which consist of a single site $x \in \Lambda$. To every polymer $P$, a (real) activity $A(P)$ is assigned. The partition function $Z(X)$ for an arbitrary finite subset $X$ of $\Lambda$ is defined as

$$
Z(X)=\sum_{X=\Sigma P_{i}} \prod_{P_{i}} A\left(P_{i}\right)
$$

Here and in the following, we write $\sum$ and + for union of disjoint sets. The sum in Eq. (2.1) runs over all partitions of $X$ into polymers. In particular

$$
Z(\emptyset)=1
$$

In our applications, the partition functions will be guaranteed to be positive, but the activities $A(P)$ for polymers other than monomers are not necessarily nonnegative. An example of a polymer system is described in Fig. 2. By definition (2.1), the partition functions satisfy the following recursion relation, for any $x \in X$

$$
Z(X)=\sum_{\substack{Y \\ Y \leqq X, x \in Y}} A(Y) Z(X-Y) .
$$

They are known as Kirkwood-Salsburg equations. Equation (2.1) may be regarded as the iterative solution of (2.2) with initial condition (2.1'). Only a finite number of iteration steps is needed to obtain $Z(X)$ for a finite set $X$.
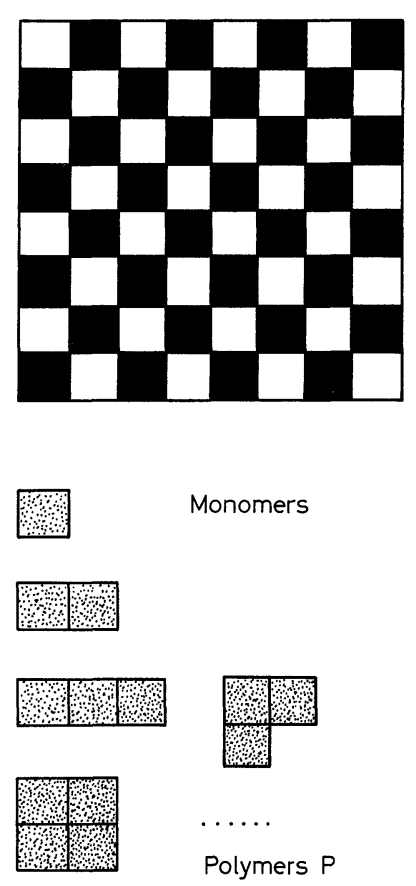

Fig. 2. Example of a polymer system. A chessboard serves as $\Lambda$ and any union of squares that can be cut out of cardboard without falling into pieces and fits on the board is a polymer. Choosing as activities $A(P)=\exp [-\beta|P|]$, the partition function would equal $e^{-64 \beta}$. (\# partitions of $\Lambda$ into polymers) 
It will be important for us that Eq. (2.1) can be inverted. That is, the activity $A(P)$ is uniquely determined by the partition functions $Z(X)$ for $X \subseteq P$. Indeed it is obvious from Eq. (2.1) that $A(P)=Z(P)-\sum \Pi$ (activities of smaller polymers). Therefore the activities can be recursively determined. The result is well known [17]

$$
A(P)=\sum_{n=1}^{|P|}(-1)^{n-1}(n-1) ! \sum_{P=\Sigma_{1}^{n} X_{i}} \prod_{i} Z\left(X_{i}\right) .
$$

The inner sum runs over partitions of $P$ into nonempty subsets $X_{i}$, and $|P|$ is the number of sites in $P$.

A function $Z(\cdot)$ of finite subsets $X \subset \Lambda$ may be regarded as a partition function of a polymer system on $\Lambda$ if it obeys Eq. (2.1). Arbitrary finite subsets of $\Lambda$ must be admitted as polymers for general $Z(\cdot)$. Since validity of Eq. (2.1) follows from (2.2) and (2.1'), it suffices to check validity of Eqs. (2.2) for some choice of $x=x(X)$ in order to verify that some $A(\cdot)$ are indeed the activities of the polymer system with partition function $Z(\cdot)$. The monomer activities $A(\{x\})=Z(\{x\})$ are positive if the partition functions are positive.

A central result of the theory of polymer systems is a series expansion for the free energies $\ln Z(X)$, together with a sufficient condition for its convergence. We state it first for finite sets $X$. Given $X$, a cluster $\mathbb{Q}$ is a nonempty collection of not necessarily distinct polymers with the property that the following graph $\gamma(\mathbb{Q})$ is connected. Draw a vertex for every polymer $P$ in $\mathbb{Q}$, and a line joining $P$ to $P^{\prime}$ if $P$ $\cap P^{\prime} \neq \emptyset$. It is customary to write

$$
\mathbb{Q}=\left(P_{1}^{n_{1}}, \ldots, P_{k}^{n_{k}}\right)
$$

if $\mathbb{Q}$ contains $k$ distinct polymers $P_{i}$ with multiplicities $n_{i} \geqq 1$. One defines reduced activities by

$$
\bar{A}(P)=A(P) / \prod_{x \in P} A(\{x\}) .
$$

The expansion formula reads

$$
\ln Z(X)=\sum_{x \in X} \ln A(\{x\})+\sum_{\mathbb{Q}} \mathfrak{a}(\mathbb{Q}) \prod_{P \in \mathbb{Q}} \bar{A}(P) .
$$

The sum over $\mathbb{Q}$ runs over all clusters of nontrivial polymers on $X$. Nontrivial polymers are, for now, all polymers other than monomers. $\mathfrak{a}(\mathbb{Q})$ are combinatorial coefficients which are given by

$$
\mathfrak{a}(\mathbb{Q})=\sum_{C \leqq \gamma(\mathbb{Q})}(-1)^{l(C)} / \prod_{i} n_{i} !
$$

Summation is over all connected subgraphs $C$ of the graph $\gamma(\mathbb{Q})$ that was mentioned above, and $l(C)$ is the number of lines in $C$. Alternative expressions and bounds on $\mathfrak{a}(\mathbb{Q})$ can be found in the literature [18].

For real $\xi>1$ one defines

$$
\mathfrak{B}_{X}(\xi)=\sup _{x \in X} \frac{1}{\xi}\left[1+\sum_{\substack{P \\ x \in P \subseteq X,|P| \geqq 2}}|\bar{A}(P)| \xi^{|P|}\right] .
$$

The expansion (2.5) for the free energy $\ln Z(X)$ converges if $\mathfrak{B}_{X}(\xi)<1$ for some $\xi>1$ $[16]$. 
By differentiating Eq. (2.5) one obtains an expansion for the so-called "reduced correlation functions" of the polymer system

$$
\varrho_{X}(Y)=\frac{Z(X-Y)}{Z(X)}=\frac{\partial}{\partial A(Y)} \ln Z(X), \quad(Y \cong X) .
$$

If $\Lambda$ is an infinitely extended lattice, then these expansions continue to converge in the infinite volume limit $X \nearrow \Lambda$, if $\mathfrak{B}_{\Lambda}(\xi)<1$ for some $\xi>1$. Moreover,

$$
\left|\varrho_{X}(Y)\right| \leqq\left[1-\mathfrak{B}_{X}(\xi)\right]^{-1} \xi^{n} \prod_{x \in Y} A(\{x\})^{-1} \text { with } n=|Y|
$$

for all $X \subseteq \Lambda$. For a more precise statement of these results, see [16]. For the renormalized Mayer expansions on the staggered lattice one uses these convergence results only at $\psi=0$. One can avoid the need to use them (in the discussion of Greens functions) altogether by imposing the renormalization condition (1.16a), viz. $\mathscr{M}(X \mid \psi=0)=0$. This can be seen from Eq. (1.19). Expansion (2.5) for $\ln Z$ serves then merely as a bookkeeping device for combinatorial factors in equations that could also be obtained by differentiating Eq. (1.12b) for $Z$. [In the expression for the connected amputated $n$-point Greens function that is obtained by differentiating expansion (2.5) with respect to the external field $\psi$ at $\psi=0$, all terms with $\mathbb{Q}$ containing more than $n$ polymers will vanish as a result of the renormalization condition (1.16a). There results a finite sum for finite $X$.] The point is that the convergence conditions mentioned above are much more restrictive than the condition for convergence of the sum (1.19) - in particular they remain nontrivial for finite $X$.

It is convenient to introduce the notion of a polymer system with empty sites. Denote its activities by $M(P)$. Its partition function is defined as

$$
Z(X)=1+\sum_{\substack{Y \\ \emptyset \subseteq X}} \sum_{Y=\Sigma P_{i}} \prod_{i} M\left(P_{i}\right) .
$$

Evidently this is equal to the partition function of a polymer system proper with activities

$$
A(\{x\})=1+M(\{x\}), \quad A(P)=M(P) \text { for }|P| \geqq 2 .
$$

We may identify with a polymer system proper in still another way. Split each site of the original set $\Lambda$ into two. Polymers of the original system, including its monomers $\{x\}$, shall occupy double sites. In addition we introduce a monomer with activity 1 which occupies only one site (either half of a double site). Applying the expansion formula (2.5) to this system we obtain

$$
\ln Z(X)=\sum_{\mathbb{Q}} \mathfrak{a}(\mathbb{Q}) \prod_{P \in \mathbb{Q}} M(P) .
$$

Summation is now over all clusters containing arbitrary polymers $P$ (of the original system). The expansion will converge if

$$
\sup _{x \in X} \frac{1}{\xi}\left[1+\sum_{x \in P}|M(P)| \xi^{2|P|}\right]<1
$$

for some $\xi>1$. Monomers are included in the sum over $P$. 
Let us now consider polymer systems whose activities depend on a field $\varphi$ on $\Lambda$

$$
\mathscr{Z}(X \mid \varphi)=\sum_{X=\Sigma P} \prod_{P} B(P \mid \varphi) .
$$

It will be required that the activity $B(P \mid \varphi)$ depends only on the restriction of $\varphi$ to $P$. We wish to consider Gaussian integrals of such partition functions. To avoid confusion with quantities that will appear later on, the polymers of the polymer system (2.14) will be called molecules, with molecular activity $B(\cdot \mid \varphi)$. Let $d \mu_{v}(\varphi)$ be the Gaussian measure with covariance $v$ (=normalized free field measure with propagator $v$ ) and consider

$$
Z(X)=\int d \mu_{v}(\varphi) \mathscr{Z}(X \mid \varphi) \equiv\langle\mathscr{Z}(X \mid \varphi)\rangle_{v} .
$$

The symbol $\langle\cdot\rangle_{v}$ stands for expectation value with respect to the Gaussian measure $d \mu_{v}$. Evidently $Z(\emptyset)=1$. We may therefore regard $Z(X)$ as partition functions of a polymer system

$$
Z(X)=\sum_{X=\Sigma P} \prod_{P} A(P) .
$$

The activities $A$ are uniquely determined by the partition functions $Z$ as we know. If $\left\{M_{1}, \ldots, M_{n}\right\}$ is a finite set of disjoint molecules, we may consider the truncated Gaussian expectation value ${ }^{5}$

$$
\left\langle B\left(M_{1} \mid \varphi\right) ; B\left(M_{2}, \varphi\right) ; \ldots ; B\left(M_{n} \mid \varphi\right)\right\rangle_{v} \equiv\left\langle\prod_{i=1}^{n}\left[B\left(M_{i} \mid \varphi\right) ;\right]\right\rangle_{v} .
$$

Truncated expectation values obey the defining relation

$$
\left\langle\prod_{a \in I} B_{a}(\varphi)\right\rangle_{v}=\sum_{I=\Sigma J} \prod_{J}\left\langle\prod_{b \in J}\left[B_{a}(\varphi) ;\right]\right\rangle_{v} .
$$

Summation is over partitions of the index set $I=\{1, \ldots, n\}$ into nonempty subsets $J$. Note that this formula has the character of a polymer representation too, with the ordinary expectation values on the left-hand side playing the role of partition functions $Z(I)$. The truncated expectation values are the corresponding activities.

It follows from Eqs. (2.15) and (2.18) that the activities $A$ in the polymer representation (2.16) of the partition functions $Z(X)$ are given by truncated expectation values

$$
A(P)=\sum_{P=\Sigma M}\left\langle\prod_{M}[B(M \mid \varphi) ;]\right\rangle_{v} .
$$

These relations can be transcribed into the language of polymer systems with empty sites. We leave this to the reader.

Truncated Gaussian expectation values admit a tree formula which expresses them as integrals over auxiliary real variables $s_{i}=0 \ldots 1$ of ordinary Gaussian expectation values. To state it we introduce some notation [19, Sect. 2.4].

An $n$-tree is a map

$$
\eta:[2, \ldots, n] \rightarrow[1, \ldots, n-1] \text { with } \eta(i)<i .
$$

5 We use the customary "semicolon notation" for truncated expectation values 
It specifies a tree graph with vertices $1 \ldots . n$, root 1 , and links $(i, \eta(i))$. Given an $(n-1)$-tupel $s=\left(s_{1}, \ldots, s_{n-1}\right)$ of real variables in the interval $0 \ldots 1$, one sets

$$
f(\eta \mid s)=\prod_{a=2}^{n} s_{a-2} s_{a-3} \ldots s_{\eta(a)}, \quad d s=d s_{1} \ldots d s_{n-1} .
$$

Empty products which arise when $\eta(a)=a-1$ (in particular for $a=2$ ) are read as 1 .

Given a set of $n$ disjoint molecules $M_{i} \subset \Lambda$, let $\chi_{i}$ be the characteristic function of $M_{i}$ (viz. $\chi_{i}(x)=1$ for $x \in M_{i}$, and $=0$ otherwise) and define the interpolating covariance $v[\pi, s]$ by

$$
v[\pi, s]=\sum_{i} \chi_{i} v \chi_{i}+\sum_{1 \leqq i<j \leqq n} s_{i} \ldots s_{j-1}\left[\chi_{\pi(i)} v \chi_{\pi(j)}+\chi_{\pi(j)} v \chi_{\pi(i)}\right] .
$$

It depends on a permutation $\pi$ of $1 \ldots n$, besides the $n-1$ tupel of real variables $s$. We assume that products of activities $B\left(M_{a} \mid \varphi\right)$ are integrable with respect to the Gaussian measure $d \mu_{v}(\varphi)$, and differentiable.

Proposition 2.1. Let $M_{1}, \ldots, M_{n}$ be disjoint subsets of $\Lambda$ and suppose that the activities $B\left(M_{a} \mid \varphi\right)$ depend only on the restriction of $\varphi$ to $M_{a}$. Then their truncated Gaussian expectation values admit the following representation in terms of ordinary Gaussian expectation values

$$
\begin{aligned}
\left\langle\prod_{a=1}^{n}\left[B\left(M_{a} \mid \varphi\right) ;\right]\right\rangle_{v}= & \sum_{\eta} \sum_{\pi}^{\prime} \int d s f(\eta \mid s) \\
& \cdot\left\langle\prod_{b=2}^{n}\left[\int_{\substack{x \in M_{\pi(b)} \\
y \in M_{\pi(n(b))}}} \frac{\delta}{\delta \varphi(x)} v(x, y) \frac{\delta}{\delta \varphi(y)}\right] \prod_{c=1}^{n} B\left(M_{c} \mid \varphi\right)\right\rangle_{v[\pi, s]} .
\end{aligned}
$$

Summation over $\eta$ is over all $n$-trees. Summation over $\pi$ is over all $(n-1)$ ! permutations of 1 ...n with $\pi(1)=1$. Integration over the $n-1$ variables $s_{i}$ runs from 0 to 1 .

This proposition is a standard result of constructive field theory $[2,19,20]$. It can be proven either by the method of partial integration of Gaussian measures of Glimm and Jaffe [19], or by making a Fourier transform in $\varphi$ and using the techniques of Mayer expansions for classical gases [20,3].

Remark. This proposition covers the special case that no two factors $B\left(M_{a} \mid \varphi\right)$ depend on the field $\varphi(x)$ at the same point $x$. A tree formula exists also in the general case where this condition is not satisfied. It can be used, for instance, to derive a tree formula for the sum of all (unrenormalized) connected $n^{\text {th }}$ order Feynman integrals, on a lattice or in the continuum. As is well known, the sum of all connected $n^{\text {th }}$ order vacuum Feynman diagrams in a theory with interaction Lagrangian $\lambda \mathscr{L}_{I}$ equals

$$
\frac{(-\lambda)^{n}}{n !} \int_{z_{1}} \ldots \int_{z_{n}}\left\langle\mathscr{L}_{I}\left(\varphi\left(z_{1}\right)\right) ; \ldots ; \mathscr{L}_{I}\left(\varphi\left(z_{n}\right)\right)\right\rangle_{v} .
$$

The general tree formula is obtained with the help of the following trick which reduces the general case to the special case covered by Proposition 2.1.

The covariance operator $v$ in a Gaussian measure is permitted to have zero eigenvalues. The simplest example is the 1-dimensional Dirac $\delta$-measure $d \mu_{0}(\xi)$ 
$=\delta(\xi) d \xi$, which is a Gaussian measure with covariance 0 . Consider the Gaussian measure for a field $\phi(x, i)$ on $\Lambda \times \ldots \times \Lambda(n$ copies $i=1 \ldots n)$ with covariance

$$
\tilde{v}(x, i, y, j)=v(x, y) \text {. }
$$

It equals

$$
d \mu_{\tilde{v}}(\phi)=d \mu_{v}(\phi(\cdot, 1)) \prod_{j=2}^{n} \prod_{x \in \Lambda} \delta(\phi(x, j)-\phi(x, 1)) d \phi(x, j) .
$$

This can be verified by checking that $\langle\phi(x, i) \phi(y, j)\rangle_{\tilde{v}}=\tilde{v}(x, i, y, j)$. Set

$$
\widetilde{B}\left(M_{i} \mid \phi\right)=B\left(M_{i} \mid \phi(\cdot, i)\right) .
$$

Evidently, for any $I \subseteq[1, \ldots, n]$,

$$
\left\langle\prod_{i \in I} B\left(M_{i} \mid \varphi\right)\right\rangle_{v}=\left\langle\prod_{i \in I} \tilde{B}\left(M_{i} \mid \phi\right)\right\rangle_{\tilde{v}} .
$$

No two activities $\widetilde{B}\left(M_{i} \mid \phi\right)$ depend on the field $\phi$ at the same point $(x, i)$, and the proposition can therefore be applied to find a formula for the truncated expectation value $\left\langle\Pi\left[B\left(M_{i} \mid \varphi\right) ;\right]\right\rangle_{v}$ in terms of a Gaussian measure for a field on $\Lambda$ $\times \ldots \times \Lambda$.

Finally we wish to consider the case that the Gaussian measure $d \mu_{v}(\varphi)$ is represented as convolution of Gaussian measures with covariances $v^{i}$ by splitting the propagator $v^{6}$. If

then

$$
v=\sum_{i=0}^{N} v^{i} \equiv v^{[\leqq N]} \quad \text { with } \quad v^{i} \geqq 0,
$$

$$
\int d \mu_{v}(\varphi) f(\varphi)=\int d \mu_{v^{0}}\left(\varphi^{0}\right) \ldots d \mu_{v^{N}}\left(\varphi^{N}\right) f\left(\sum_{i=0}^{N} \varphi^{i}\right) .
$$

Gaussian measures are only well defined for propagators $v$ that are kernels of positive semidefinite operators. Therefore the split of the propagator must be such that $v^{i}$ have this property, viz. $v^{i} \geqq 0$ (as operators).

Let us first give the result for a split of a propagator $v$ into two. The general case will then be handled by iterating the result.

Proposition 2.2. Let $v=u+w$ with $v \geqq 0, u \geqq 0$ and $w \geqq 0$ as operators. Then the truncated expectation values obey the following relations

$$
\left\langle\prod_{a \in I}\left[B_{a}(\varphi) ;\right]\right\rangle_{v}=\sum_{I=\Sigma J}\left\langle\prod_{J}\left[\left\langle\prod_{b \in J}\left[B_{b}(\Phi+\zeta) ;\right]\right\rangle_{w} ;\right]\right\rangle_{u} .
$$

The expectation value $\langle\cdot\rangle_{w}$ is computed with Gaussian measure $d \mu_{w}(\zeta)$, and the expectation value $\langle\cdot\rangle_{u}$ with $d \mu_{u}(\Phi)$. Summation is over partitions of the finite set I into nonempty subsets $J \cong I$.

The proof of this proposition will be given below, at the end of this section.

6 The material in the rest of this section is not needed for pedestrian calculations 
Let us now iterate Eq. (2.29) to treat the split (2.27) of the propagator and corresponding split of the field in (2.28). Write

$$
\begin{aligned}
& v \equiv v^{[\leqq N]}=v^{N}+v^{[\leqq N-1]}=\ldots=v^{[>N-\ell]}+v^{[\leqq N-\ell]}, \\
& v^{[\leqq N-1]}=v^{N-1}+v^{[\leqq N-2]}
\end{aligned}
$$

and

$$
\begin{aligned}
\varphi \equiv \varphi^{[\leqq N]}=\varphi^{N}+\varphi^{[\leqq N-1]}=\ldots=\varphi^{[>N-\ell]}+\varphi^{[\leqq N-\ell]}, \\
\varphi^{[\leqq N-1]}=\varphi^{N-1}+\varphi^{[\leqq N-2]}
\end{aligned}
$$

The labelling of the propagators shall be such that $v^{N}$ propagates the highest frequencies, and $v^{0}$ the lowest. (It was the other way around in [4].) Following $[3,4]$ we define $\ell$-vertices with constituents $i \in I$ and associated vertex functions inductively, for a given index set $I \leqq N$. A 0 -vertex is a single element $a \in I$ which is its own constituent. The associated vertex function is

$$
\sigma_{a}^{0}\left(\varphi^{[\leqq N]}\right)=B\left(M_{a} \mid \varphi^{[\leqq N]}\right) .
$$

Higher vertices are defined inductively. An $\ell$-vertex $\alpha^{\prime}$ is a finite collection $\{\alpha\}$ of $(\ell-1)$-vertices, no two of which share a constituent. $a \in I$ is a constituent of $\alpha^{\prime}$ if it is a constituent of one of the $(\ell-1)$-vertices $\alpha \in \alpha^{\prime}$. We write $a € \alpha^{\prime}$ in this case, and $C\left(\alpha^{\prime}\right)$ for the set of all constituents of $\alpha^{\prime}$. The vertex functions are defined by the recursion relation

$$
\sigma_{\alpha^{\prime}}^{\ell}\left(\varphi^{[\leqq N-\ell]}\right)=\left\langle\prod_{\alpha \in \alpha^{\prime}}\left[\sigma_{\alpha}^{\ell-1}\left(\varphi^{[\leqq N-\ell]}+\varphi^{N-\ell+1}\right) ;\right]\right\rangle_{v^{N-\ell+1}} .
$$

The truncated expectation value is computed with Gaussian measure $d \mu_{v^{N-\ell+1}}\left(\varphi^{N-\ell+1}\right)$. It follows from Proposition 2.2 by induction that

$$
\left\langle\prod_{b \in J}\left[B\left(M_{b} \mid \psi+\varphi^{[>N-\ell]}\right) ;\right]\right\rangle_{v^{[>N-\ell]}}=\sum_{\substack{\alpha \\ C(\alpha)=J}} \sigma_{\alpha}^{\ell}(\psi)
$$

for all $\ell=0,1, \ldots, N$. Summation is over all $\ell$-vertices $\alpha$ whose set of constituents is $C(\alpha)$. The special case $\ell=N+1$ gives

$$
\left\langle\prod_{a \in I}\left[B\left(M_{a} \mid \varphi\right) ;\right]\right\rangle_{v}=\sum_{\substack{\alpha \\ C(\alpha)=I}} \sigma_{\alpha}^{N+1}(0) .
$$

Summation is over all $(N+1)$-vertices with set of constituents $I$. (The present definition of vertex functions differs by a combinatorial factor from $[3,4]$.) Following Gallavotti and Nicoló [12], the terms in the sums (2.33) or (2.34) [i.e. the $(N+1)$-vertex $\alpha]$ can be represented by tree diagrams. To avoid confusion with other kinds of trees that occurred before, we call them Gallavotti Nicoló trees (GN-trees).

The trees are drawn on ruled paper, with lines labelled by $k=-1, \ldots, N$. A $\ell$-vertex $\alpha$ is represented by a point on line $N-\ell$. If the $\ell$-vertex $\alpha$ consists of $(\ell-1)$-vertices $\alpha_{1}, \ldots, \alpha_{k}$, then the point $\alpha$ on line $N-\ell$ is linked to $k$ points $\alpha_{1}, \ldots, \alpha_{k}$ 


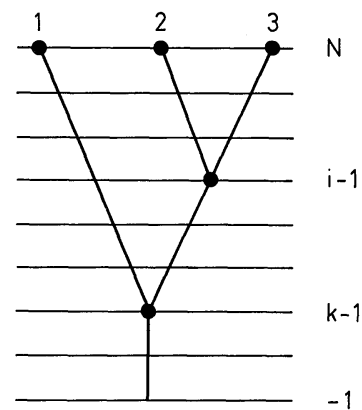

Fig. 3. Example of a Gallavotti-Nicoló tree. The tree shown in the figure represents the expectation value

$$
\left\langle\left\langle B\left(M_{1}, \varphi\right) ;\left\langle B\left(M_{2}, \varphi\right) ; B\left(M_{3}, \varphi\right)\right\rangle_{v^{i}}\right\rangle_{v^{k}}\right.
$$

assuming $M_{1}, M_{2}, M_{3}$ consist of points of $\Lambda_{i}$ and $\Lambda_{k}$ only

on line $N-\ell+1$. Then the procedure is repeated. If the $(\ell-1)$-vertex $\alpha_{1}$ consists of $(\ell-2)$-vertices $\alpha_{11}, \ldots, \alpha_{1 m}$, it is linked to $m$ points $\alpha_{1 i}$ on line $N-\ell+2$, etc. If $\alpha$ had constituent set $I$ then the procedure ends with the drawing of points on line $N$ representing 0 -vertices, one for each constituent $=$ element of $I$.

In this way, a one to one correspondence is established between $\ell$-vertices and GN trees whose root is on line $N-\ell$, and whose tips of branches on line $N$ are labelled by the elements of the constituent set $C(\alpha)$. In our applications it happens often that the activities $B\left(M_{a} \mid \varphi\right)$ are all independent of some $\varphi^{j}$ so that the integration $\int d \mu_{v^{j}}\left(\varphi^{j}\right)$ is trivial. In this case the GN-tree cannot branch on line $j-1$, and we erase the vertices on this line. An example is shown in Fig. 3.

These formulae embody the essence of the iterated Mayer expansions of $[3,4]$ in a succinct way. They are also the starting point of the perturbative renormalization theory of Gallavotti and Nicoló [12]. The truncated expectation values in the right-hand side of recursion relation (2.32) can be expressed in a tree formula by Proposition 2.1 (and remark following it).

It remains to prove Proposition 2.2. Since truncated expectation values are uniquely determined by ordinary expectation values by the defining relation (2.18), it suffices to show that

$$
\left\langle\prod_{a \in H} B_{a}(\varphi)\right\rangle_{w+u}=\sum_{H=\Sigma K} \prod_{K}\left\{\sum_{K=\Sigma J}\left\langle\prod_{J}\left[\left\langle\prod_{b \in J}\left[B_{b}(\Phi+\zeta) ;\right]\right\rangle_{w}\right] ;\right\rangle_{u}\right\} \cdot
$$

By Eq. (2.28) the left-hand side equals

$$
\left\langle\left\langle\prod_{a \in \boldsymbol{H}} B_{a}(\Phi+\zeta)\right\rangle_{w}\right\rangle_{u}
$$

By the defining relation (2.18) of truncated expectation values this equals

$$
\sum_{H=\Sigma J}\left\langle\prod_{J}\left\langle\prod_{b \in J}\left[B_{b}(\Phi+\zeta) ;\right]\right\rangle_{w}\right\rangle_{u}
$$

Using the defining relation (2.18) of truncated expectation values again to reexpress the outer ordinary expectation value, this becomes

$$
\sum_{H=\Sigma J} \sum_{\{J\}=\Sigma \mathscr{K}} \prod_{\mathscr{K}}\left\langle\prod_{J \in \mathscr{K}}\left[\left\langle\prod_{b \in J}\left[B_{b}(\Phi+\zeta) ;\right]\right\rangle_{w} ;\right]\right\rangle_{u} .
$$


The inner sum is over partition of the set $\{J\}$ (of subsets of $H$ ) into disjoint subsets $\mathscr{K}$. Thus, $\mathscr{K}$ is a collection of sets $J$. Set $K=\sum_{J \in \mathscr{K}} J$. Then

$$
\sum_{H=\Sigma J} \sum_{\{J\}=\Sigma \mathscr{K}} \prod_{\mathscr{K}}\left\langle\prod_{J \in \mathscr{K}} \cdot\right\rangle=\sum_{H=\Sigma K} \prod_{K}\left\{\sum_{K=\Sigma J}\left\langle\prod_{J} \cdot\right\rangle\right\}
$$

by the distributive law. Upon inserting this, expression (2.36) becomes equal to the right-hand side of Eq. (2.35). q.e.d.

\section{Expansions on the Staggered Lattice}

Now we consider $\lambda \phi^{4}$-theory in $v=3$ dimensions, in the continuum or on a cubic lattice of arbitrarily small lattice spacing $a \equiv a_{N}$. This space, on which the theory lives originally, will be called the base space. Integration over the base space is written as

$$
\int_{z \in \text { base }} \equiv \begin{cases}\int_{\mathbb{R}^{3}} d^{3} z & \text { in the continuum } \\ a^{3} \sum_{z \in(a \mathbb{Z})^{3}} & \text { on the lattice. }\end{cases}
$$

The generating function for amputated Greens functions, the Mayer amplitudes etc., will depend on an external field $\psi$ on base space.

We put the theory on a staggered lattice $\Lambda$ as described in the introduction. When the interaction and induced counterterms are switched off outside a finite subset $X$ of $\Lambda$, the partition function becomes

$$
Z(X \mid \psi) \equiv Z_{1}(X \mid \psi) \exp \left[-\frac{\lambda}{4 !} \int_{\text {base }} \psi(z)^{4}\right]=\int d \mu_{v_{X}}(\varphi) \mathscr{Z}(X \mid \psi+\mathscr{A} \varphi) .
$$

Integration is over fields $\varphi$ that vanish outside $X$, so that the integral has as many dimensions as $X$ has points, and

$$
\mathscr{Z}(X \mid \psi)=\exp \left\{\int_{z \in \text { base }}\left[-\frac{\lambda}{4 !} \psi(z)^{4}+\frac{1}{2} \delta m^{2}\left(v_{X} \mid z\right) \psi(z)^{2}\right]+\delta e(X)\right\} .
$$

The mass counterterm is given by Eq. (1.4), and the vacuum energy counterterm $\delta e(X) \equiv \delta e\left(v_{X}\right)$ can be determined so that

$$
Z(X \mid \psi=0)=1 .
$$

The factor $\exp \left[-\lambda \int \psi^{4} / 4 !\right]$ is extracted in (3.2) in order to assure that $Z_{1}(\emptyset \mid \psi)=1$, as is necessary for partition functions of polymer systems by Eq. (2.1).

The Mayer amplitudes $\mathscr{M}$ are related to the partition functions.

$$
\begin{aligned}
Z_{1}(X \mid \psi) & =\sum_{X=\Sigma Y} \prod_{Y} A(Y \mid \psi), \\
A(X \mid \psi) & =\delta_{1, n}+M(X \mid \psi), \\
M(X \mid \psi) & =n ! a\left(x_{1}\right)^{v} \ldots a\left(x_{n}\right)^{v} \mathscr{M}\left(x_{1}, \ldots, x_{n} \mid \psi\right), \quad(v=3)
\end{aligned}
$$

for $X=\left\{x_{1}, \ldots, x_{n}\right\}$ with $a(x)=a_{j}$ for $x \in \Lambda_{j}$. A are the activities of the polymer system whose partition functions are $Z_{1}$, and $M$ are the activities of the 
corresponding polymer system with empty sites. Arbitrary finite nonempty subsets of the staggered lattice are admitted as polymers. According to the discussion in Sect. 2, activities are uniquely determined by partition functions for polymer systems. Therefore the Mayer amplitudes are well defined for distinct arguments $x_{1}, \ldots, x_{n}$. They are symmetrical in these arguments.

It is useful to introduce also augmented Mayer emplitudes $\tilde{M}\left(x_{1}, \ldots, x_{n} \mid \psi\right)$. They are defined for arbitrary, not necessarily distinct, values of their arguments, and symmetrical in these arguments. If all arguments $x_{i}$ are distinct

$$
\tilde{M}\left(x_{1}, \ldots, x_{n} \mid \psi\right)=\mathscr{M}\left(x_{1}, \ldots, x_{n} \mid \psi\right) \quad\left(x_{i} \text { distinct }\right) \text {. }
$$

The general definition is as follows. Consider clusters $\mathbb{Q}$ of polymers $P \subset A$ (including monomers) as defined in Sect. 2. Let $\operatorname{supp} \mathbb{Q}$ be the disjoint union of $P \in \mathbb{Q}$ - i.e. a point $x \in \Lambda$ is contained in supp $\mathbb{Q}$ with a certain multiplicity $n(x)$. It equals the number of polymers $P \in \mathbb{Q}$ which contain $x$. Now define, for $X=$ finite family of not necessarily distinct elements $x_{i}$ of $\Lambda$

$$
\begin{gathered}
\tilde{M}(X \mid \psi)=\sum_{\substack{\mathbb{Q} \\
\text { supp } \mathbb{Q}=X}} \mathfrak{a}(\mathbb{Q}) \prod_{P \in \mathbb{Q}} M(P \mid \psi), \\
n ! a\left(x_{1}\right)^{v} \ldots a\left(x_{n}\right)^{v} \tilde{\mathscr{M}}\left(x_{1}, \ldots, x_{n} \mid \psi\right)=\tilde{M}(X \mid \psi) \prod_{\substack{\text { distinct } \\
x \in X}} n(x) !
\end{gathered}
$$

for $X=\left\{x_{1}, \ldots, x_{n}\right\}$. The combinatorial factors $\mathfrak{a}(\mathbb{Q})$ were defined by Eq. (2.6). Equation (3.6a) is a special case of $(3.6 \mathrm{~b}, \mathrm{c})$ because $\mathbb{Q}$ consists of a single polymer $X$ if $\operatorname{supp} \mathbb{Q}$ contain no point with multiplicity $\geqq 2$, and $\mathfrak{a}(\mathbb{Q})=1$ in this case. In general, the total number of arguments $x \in P$ in the amplitudes $M$ on the righthand side of $(3.6 \mathrm{~b})$ is $n$, and the sum over clusters $\mathbb{Q}$ is a finite sum.

It follows from Eqs. (2.12) and (3.6b) that

$$
\ln Z_{1}(X \mid \psi)=\sum_{X} \tilde{M}(X \mid \psi)=\sum_{n \geqq 1} \int_{x_{1}} \ldots \int_{x_{n} \in X} \tilde{\mathscr{M}}\left(x_{1}, \ldots, x_{n} \mid \psi\right)
$$

in the sense of formal power series in the amplitudes $\mathscr{M}(\ldots)$. The sum is actually convergent if the Mayer amplitudes satisfy suitable bounds. For the computation of Greens functions this is not needed when the normalization condition (3.4) is imposed, and $\psi$ may be considered as infinitesimal in this case. See the discussion in Sect. 2.

Now we turn to the computation of activities $A$ (or Mayer amplitudes $\mathscr{M}$ ). Write $\varphi_{X}$ for a field that vanishes outside $X$ and set

$$
\mathscr{Z}\left(X \mid \psi+\mathscr{A} \varphi_{X}\right)=\mathscr{Z}_{1}\left(X \mid \psi, \varphi_{X}\right) \exp \left[-\frac{\lambda}{4 !} \int_{\text {base }} \psi(z)^{4}\right]
$$

so that $\mathscr{Z}_{1}(\emptyset \mid \psi, \cdot)=1$. We regard $\mathscr{Z}_{1}\left(X \mid \psi, \varphi_{X}\right)$ as partition function of a polymer system.

$$
\mathscr{Z}_{1}\left(X \mid \psi, \varphi_{X}\right)=\sum_{X=\Sigma P} \prod_{P} B\left(P \mid \psi, \varphi_{P}\right) \quad \text { with } \quad \varphi_{P}(x)= \begin{cases}\varphi_{X}(x) & \text { for } x \in P \subseteq X \\ 0 & \text { otherwise }\end{cases}
$$

The polymers of this polymer system will be called molecules, and their activities $B$ will be called molecular activities. Arbitrary finite nonempty subsets of $\Lambda$ are 
admitted as molecules. A pedestrian way to compute the molecular activities from the given interaction (3.3) uses the inversion formula (2.3) viz.

$$
B\left(P \mid \psi, \varphi_{P}\right)=\sum_{n \geqq 1}(-1)^{n-1}(n-1) ! \sum_{P=\Sigma_{1}^{n} X_{i}} \mathscr{Z}_{1}\left(X_{i} \mid \psi, \varphi_{X_{i}}\right) .
$$

A more convenient formula will be derived later on.

By inserting Eqs. (3.8), (3.9) into (3.2), $Z_{1}(X \mid \psi)$ becomes expressed as a Gaussian expectation of a partition function $\mathscr{Z}_{1}(X, \cdot \cdot)$ of a polymer system. According to the discussion in Sect. 2 [Eq. (2.19)] this implies that the corresponding activities are obtained as truncated expectation values

$$
A(X \mid \psi)=\sum_{X=\Sigma P}\left\langle\prod_{P}\left[B\left(P \mid \psi, \varphi_{P}\right) ;\right]\right\rangle_{v_{X}} .
$$

The Mayer amplitudes are given in terms of $A$ 's by Eqs. $(3.5 \mathrm{~b}, \mathrm{c})$. The truncated expectation values can be expressed in terms of ordinary expectation values by the tree formula, Proposition 2.1. Finally we note again that the resulting Gaussian integrals are $n$-dimensional integrals if $X$ has $n$ points. Let us elaborate on this point.

Let $X$ be a subset containing $n$ elements of $\Lambda$, and suppose that the function $F$ of the field $\varphi$ depends only on $\varphi(x)$ for $x \in X$. Set

$$
\varphi_{x}=a_{j}^{d} \varphi(x) \text { for } x \in X \cap \Lambda_{j}, \text { with } d=\frac{1}{2}(v-2)=\frac{1}{2},
$$

and consider the $n \times n$ matrix

$$
\begin{gathered}
\mathbb{V}=\left(\mathbb{V}_{x y}\right)_{x, y \in X} \\
\mathbb{V}_{x y}=a_{j}^{2 d} v(x, y) \text { for } x \in X \cap \Lambda_{j}
\end{gathered}
$$

$\mathbb{V}_{x y}$ is zero unless both $x$ and $y$ are in the same layer $X \cap \Delta_{j}$. By hypothesis

$$
F(\varphi)=f\left(\left\{\varphi_{x}\right\}_{x \in X}\right) .
$$

In this notation

$$
\int d \mu_{v}(\varphi) F(\varphi)=(\operatorname{det} 2 \pi \mathbb{V})^{-\frac{1}{2}} \int\left[\prod_{x \in X} d \varphi_{x}\right] e^{-\frac{1}{2} \Sigma_{x, y \in X} \varphi_{x}\left(\mathbb{V}^{-1}\right)_{x y} \varphi_{y}} f\left(\left\{\varphi_{z}\right\}\right) .
$$

The right-hand side of this formula is an $n$-dimensional integral as promised. Equation (3.15) can be verified by using the standard formula for the Fourier transform of a Gaussian measure

$$
\int d \mu_{v}(\varphi) e^{i(q, \varphi)}=e^{-\frac{1}{2}(q, v q)} .
$$

Instead of using the explicit formula (3.10) for the molecular activities $B$ in terms of the interaction, they can also be determined from a recursion relation which we will now describe. Let us set

$$
\mathscr{Z}^{Y}\left(X \mid \psi+\mathscr{A} \varphi_{X}\right)=\mathscr{Z}\left(X+Y \mid \psi+\mathscr{A} \varphi_{X}\right) \quad \text { for } \quad X \cap Y=\emptyset .
$$

The quantity $\mathscr{Z}_{2}^{Y}(X)=\mathscr{Z}^{Y}(X) / \mathscr{Z}^{Y}(\emptyset)$ obeys $\mathscr{Z}_{2}^{Y}(\emptyset)=1$ and may therefore be regarded as partition function of a polymer system. Denote the corresponding activities by $B^{Y}\left(P \mid \psi, \varphi_{P}\right)$. They obey the Kirkwood-Salsburg equations [for any 


$$
\begin{aligned}
x=x(P) \in P] & \\
& \mathscr{Z}^{Y}\left(X \mid \psi+\mathscr{A} \varphi_{X}\right)=\sum_{\substack{P \\
x \in P \subseteq X}} B^{Y}\left(P \mid \psi, \varphi_{P}\right) \mathscr{Z}^{Y}\left(X-P \mid \psi+\mathscr{A} \varphi_{X-P}\right) .
\end{aligned}
$$

The molecular activities in which we are actually interested (for now) are

$$
B\left(P \mid \psi, \varphi_{P}\right)=B^{\emptyset}\left(P \mid \psi, \varphi_{P}\right) .
$$

Let us write $P+x$ in place of $P \cup\{x\}$ if $x \notin P$, and $\varphi_{x}$ in place of $\varphi_{\{x\}}$. $\varphi_{P}$ is short for $\chi_{P} \varphi, \chi_{P}=$ characteristic function of $P \subset \Lambda$.

Proposition 3.1. The generalized molecular activities obey the following recursion relation for $w \notin P+Y, x \in P$

$$
\begin{aligned}
B^{Y}\left(P+w \mid \psi, \varphi_{P+w}\right)= & \sum_{\substack{R \\
x \in R \cong P}} B^{Y}\left(P-R+w \mid \psi, \varphi_{P-R+w}\right) \\
& \cdot\left[B^{Y+w}\left(P \mid \psi+\mathscr{A} \varphi_{w}, \varphi_{P}\right)-B^{Y}\left(P \mid \psi, \varphi_{P}\right)\right],
\end{aligned}
$$

and the initial condition

$$
B^{Y}\left(\{x\} \mid \psi, \varphi_{x}\right)=\mathscr{Z}\left(Y+x \mid \psi+\mathscr{A} \varphi_{x}\right) / \mathscr{Z}(Y \mid \psi) .
$$

The notation $\mathscr{A} \varphi$ was introduced in Eq. (1.5); the other quantities were introduced earlier in this section.

The proof of Proposition 3.1 is given in Appendix C. This recursion relation can be solved by methods similar to those of Sect. 2. We shall defer their further analysis to Part II and record here only one result.

Corollary 3.2. If $P$ consists of $n$ points, $4 k-3 \leqq n \leqq 4 k$, then

$$
B\left(P \mid \psi, \varphi_{P}\right)=\delta_{1, n}+O\left(\lambda^{k}\right) .
$$

It follows from Eq. (3.11) that the activities $A(X \mid \psi)$ share this property. Let us summarize the results obtained so far as

Theorem 3.3. Let the molecular activities $B\left(P \mid \psi, \varphi_{P}\right) \equiv B^{\emptyset}\left(P \mid \psi, \varphi_{P}\right)$ be determined by the interaction Boltzmannian

$$
\mathscr{Z}(X \mid \psi)=\exp \left\{\delta e(X)+\int_{z \in \text { base }}\left[-\frac{\lambda}{4 !} \psi(z)^{4}+\frac{1}{2} \delta m^{2}\left(v_{X} \mid z\right) \psi(z)^{2}\right]\right\}
$$

via Eqs. (3.8), (3.10) or Proposition 3.1. Then the Mayer amplitudes are expressed as truncated expectation values as follows. Let $X=\left\{x_{1}, \ldots, x_{n}\right\}$. Then

$$
\begin{gathered}
n ! a\left(x_{1}\right)^{v} \ldots a\left(x_{n}\right)^{v} \mathscr{M}\left(x_{1}, \ldots, x_{n} \mid \psi\right)=A(X \mid \psi)-\delta_{1, n}, \\
A(X \mid \psi)=\sum_{X=\Sigma P}\left\langle\prod_{P}\left[B\left(P \mid \psi, \varphi_{P}\right) ;\right]\right\rangle_{v_{X}} .
\end{gathered}
$$

The truncated expectation value is expressed in terms of n-dimensional Gaussian integrals by Proposition 2.1 and Eq. (3.15). If $4 k-3 \leqq n \leqq 4 k$ then

$$
\mathscr{M}\left(x_{1}, \ldots, x_{n} \mid \psi\right)=O\left(\lambda^{k}\right) \text {. }
$$


The generating function for connected free-propagator-amputated Greens functions is expressed in terms of Mayer amplitudes by Eqs. (1.1) and (3.6). Alternatively, the full (disconnected) free-propagator-amputated Greens functions may be obtained from Eqs. (1.19).

Let us note that the tree formula (Proposition 2.1) involves also integrations over $n-1$ real variables $s_{i}=0 \ldots 1$, and summation over $n-1$ integers $\eta(a)=1 \ldots a-1$ (apart from symmetrization $\pi$ ). They replace summation over all Feynman graphs in standard perturbation theory. The summation over order $n$ and arguments $x_{1}, \ldots, x_{n}$ in Eqs. (1.1) or (1.19) for the Greens functions replace the sum over orders and integrals over coordinate space in standard perturbation theory.

If one wishes to, one can compute the truncated expectation value (3.11) integrating out the fields $\varphi^{j}$ on $\Lambda_{j}$ in sequence, beginning with the highest frequencies $j$. How this is done is explained at the end of Sect. 2. The result is given by a sum of terms that are labelled by Gallavotti-Nicoló trees. The tree formula (Proposition 2.1) can be used to do the Gaussian integration in the recursion relation for vertex functions, Eq. (2.32).

Of course, the truncated expectation values (3.11) can also be evaluated without recourse to the tree formula (Proposition 2.1) by using their standard expression in terms of ordinary expectation values, and Eqs. (3.8), (3.10) can be used in place of the recursion relation (Proposition 3.1).

In Eq. (3.11) the renormalization cancellation occurs between different terms in the sum over partition $X=\sum P$ of $X$. In Part II we will show how to change this by giving the same $s$-dependence to the propagators that appear as arguments of counterterms and to the covariance of the Gaussian measure in the tree formula.

\section{Relation with Perturbation Theory}

The Mayer amplitudes may be expanded in formal (asymptotic) power series in the coupling constant $\lambda$. Theorem 3.3 asserts that $\mathscr{M}\left(x_{1}, \ldots, x_{n} \mid \psi\right)$ is of order $\lambda^{k}$ if $4 k-3 \leqq n \leqq 4 k$. It follows from their definition (3.6) that the same is then also true for the augmented Mayer amplitudes $\tilde{\mathscr{M}}\left(x_{1}, \ldots, x_{n} \mid \psi\right)$. Therefore, if we truncate the series (1.1) for the generating function of the connected free-propagatoramputated Greens functions after the term $n=4 k$, the result will be correct to order $\lambda^{k}$. In other words, the result differs from the sum of all Feynman diagrams up to order $k$ by higher order and nonperturbative contributions.

In order to deepen the reader's understanding of this fact we will now indulge in a pedagogical exercise. We will examine $v$-dimensional $\lambda \phi^{4}$-theory without any counterterms on a lattice $A$ of lattice spacing $a$, and its Mayer expansions on this simple lattice. This may be regarded as a simple special case of the general one-the staggered lattice has only one layer, and the counterterms are zero.

We will exhibit an explicit relation between the Mayer amplitudes, expanded in a formal power series in $\lambda$, and the Feynman amplitudes. Stated in words it says that the Mayer amplitude $\mathscr{M}\left(x_{1}, \ldots, x_{n} \mid \psi\right)$ equals the sum of all "point connected" Feynman diagrams whose arbitrarily many vertices occupy the $n$ distinct points $x_{1}, \ldots, x_{n}$ of the lattice $\Lambda$. A Feynman diagram with given positions of its vertices is 
called point connected if it is connected or becomes connected when vertices that are positioned at the same site of the lattice are identified.

The partition function at zero external field for the simplified model is

$$
Z(\Lambda \mid 0)=\int d \mu_{\nu}(\phi) \exp \left[-\frac{\lambda}{4 !} \int_{z \in \Lambda} \phi(z)^{4}\right] .
$$

In perturbation theory, the partition function is obtained as a sum of coordinate space integrals associated with arbitrary not necessarily connected vacuum Feynman diagrams.

$Z(\Lambda \mid 0)=\sum$ (disconnected vacuum Feynman diagrams)

$$
=1+\int_{x_{1}} Q_{x_{1}}+\int_{x_{1}} \int_{x_{2}}\left[\bigotimes_{x_{2}}+\varnothing+\underset{Q}{Q}\right]+\ldots .
$$

We adopt the convention that Feynman integrands associated with arbitrary diagrams are to be evaluated according to the standard rules, including combinatorial factors. The combinatorial factors coming from symmetries under permutations of disconnected pieces imply, for instance

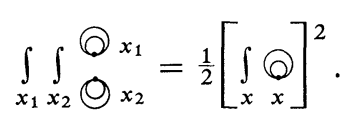

As a result of these combinatorial factors, the series of $Z(\Lambda \mid 0)$ exponentiates to a sum of integrals associated with connected vacuum diagrams,

$\ln Z(\Lambda \mid 0)=\sum$ (connected vacuum diagrams)

$$
=\int_{x_{1}} \underset{x_{1}}{Q}+\int_{x_{1}} \int_{x_{2}}\left[\bigoplus_{x_{2}}^{x_{1}}+\underset{Q}{Q}\right]+\ldots .
$$

Now we perform a reordering and partial resummation. In (4.2) we collect all the terms in the combined sum, over graphs and over $n$-tuples of points $\left(x_{1}, \ldots, x_{k}\right)$ with arbitrary $k \geqq n$, whose vertices $\left\{x_{i}\right\}_{i=1 \ldots k}$ occupy the same set $Y=\left\{y_{1}, \ldots, y_{n}\right\} \subseteq \Lambda$ of $n$ distinct lattice points.

$$
\begin{aligned}
Z(\Lambda \mid 0)= & 1+\int_{x_{1}}\left[Q+a^{v}\left\{\S x_{1}+\infty 0+\delta\right\}+\ldots\right] \\
& +\int_{x_{1} \neq x_{2}}\left[\bigcirc_{x_{2}}^{x_{1}}+8+Q+\ldots\right] \\
& +\ldots \\
\equiv & 1+\int_{x_{1}} \mathscr{M}_{1}\left(x_{1}\right)+\iint_{x_{1} \neq x_{2}}\left[\mathscr{M}_{2}\left(x_{1}, x_{2}\right)+\frac{1}{2} \mathscr{M}_{1}\left(x_{1}\right) \mathscr{M}_{1}\left(x_{2}\right)\right]+\ldots
\end{aligned}
$$

etc., $\mathscr{M}_{n}\left(x_{1}, \ldots, x_{n}\right)$ is the sum of all point connected vacuum Feynman diagrams whose vertices are positioned at $n$ distinct sites $y_{1}, \ldots, y_{n}$ of the lattice. There are infinitely many such diagrams because arbitrarily many vertices may be positioned 
on the same site $x$. In effect we are summing up diagrams with arbitrarily many propagators joining any two (identical or distinct) sites $y_{1}, \ldots, y_{n}$ of the lattice.

By construction, $\mathscr{M}_{n}\left(x_{1}, \ldots, x_{n}\right)$ will be of order $\lambda^{n}$ as $\lambda \rightarrow 0$. An expansion for $\ln Z(\Lambda \mid 0)$ is obtained from the expansion for $Z(\Lambda \mid 0)$ by multiplying $\mathscr{M}_{n}$ with $\xi^{n}$, expanding in powers of $\xi$ and setting $\xi=1$ in the end. This expansion will include correction terms that come from the constraints $x_{1} \neq x_{2}$ etc. in the sum for $Z(\Lambda \mid 0)$ ("excluded volume effect").

$$
\begin{aligned}
\ln Z(\Lambda \mid 0)= & \int_{x_{1}} \mathscr{M}_{1}\left(x_{1}\right)+\iint_{x_{1} \neq x_{2}} \mathscr{M}_{2}\left(x_{1}, x_{2}\right)+\ldots \\
& -\frac{1}{2} a^{v} \int_{x_{1}} \mathscr{M}_{1}\left(x_{1}\right)^{2}-\ldots \\
& \pm \ldots \\
= & \int_{x_{1}} \tilde{\mathscr{M}}_{1}\left(x_{1}\right)+\iint_{x_{1} x_{2}} \tilde{\mathscr{M}}_{2}\left(x_{1}, x_{2}\right)+\ldots
\end{aligned}
$$

with

$$
\begin{aligned}
\tilde{\mathscr{M}}_{1}\left(x_{1}\right) & =\mathscr{M}_{1}\left(x_{1}\right) \\
\tilde{M}_{2}\left(x_{1}, x_{2}\right) & =\left\{\begin{array}{lll}
\mathscr{M}_{2}\left(x_{1}, x_{2}\right) & \text { if } & x_{1} \neq x_{2} \\
-\frac{1}{2} \mathscr{M}_{1}\left(x_{1}\right)^{2} & \text { if } & x_{1}=x_{2} .
\end{array}\right.
\end{aligned}
$$

The whole discussion can be repeated for $Z(\Lambda \mid \psi)$ with general external field $\psi$ (see Appendix A). One starts from

$$
Z(\Lambda \mid \psi)=1+\int_{x_{1}}\left[Q+\underset{\psi}{Q}+{ }_{\psi}^{\psi} \ddots_{\psi}^{\psi}\right]+\ldots
$$

The Mayer amplitudes $\mathscr{M}_{n}\left(x_{1}, \ldots, x_{n}\right)$ will now depend on $\psi$ through $\psi\left(x_{1}\right), \ldots, \psi\left(x_{n}\right)$. The general form of the expansion for $Z(\Lambda \mid \psi)$ is

$$
\begin{gathered}
Z(\Lambda \mid \psi)=1+\sum_{N \geqq 1} \sum_{k} \sum_{\substack{\left\{\ell_{j}\right\}_{1}^{k} \\
\Sigma \ell_{j}=N}} c\left(\left\{l_{j}\right\}\right) \int_{\substack{x_{1} \\
\text { distinct }}} \ldots \int_{x_{n}} \mathscr{M}_{\ell_{1}}\left(x_{1}, \ldots, x_{\ell_{1}}\right) \\
\cdot \mathscr{M}_{\ell_{2}}\left(x_{\ell_{1}+1}, \ldots, x_{\ell_{1}+\ell_{2}}\right) \ldots \mathscr{M}_{\ell_{k}}\left(\ldots x_{N}\right) .
\end{gathered}
$$

The combinatorial factor is

$$
c\left(\left\{\ell_{j}\right\}\right)=\prod_{\ell=1}^{\infty} \frac{1}{m_{\ell} !}
$$

if $m_{\ell}$ of the integers $\ell_{j} \geqq 1$ equal $\ell$.

Let us state the relation between Mayer amplitudes and connected Feynman diagrams in general terms.

Consider $n \times \ell$ matrices $k^{(\ell)}=\left(k_{i j}^{(\ell)}\right)$ whose entries are nonnegative integers. By a permutation of rows or columns, such a matrix can be brought to block form. We call it irreducible if it consists of only one block and no row or column is identically 0 . We write $\mathscr{F}\left(y_{1}, \ldots, y_{k}\right)$ for the sum of all connected $k^{\text {th }}$ order vacuum diagrams with vertices positioned at $y_{1}, \ldots, y_{k}$. We abbreviate

$$
\mathscr{F}(\underbrace{x_{1}, \ldots, x_{1}}_{m_{1}}, x_{2} \ldots, \underbrace{x_{n}, \ldots, x_{n}}_{\text {arguments }}) \equiv \mathscr{F}\left(x_{1}^{m_{1}}, \ldots, x_{n}^{m_{n}}\right) .
$$

We shall not indicate the dependence on the external field $\psi$ explicitly. 
Proposition 4.1.

$$
\mathscr{M}_{n}\left(x_{1}, \ldots, x_{n}\right)=\frac{1}{n !} \sum_{\ell \geqq 1} \sum_{k^{(\ell)}} \frac{\prod_{j=1}^{\ell}\left(\sum_{i=1}^{n} k_{i j}\right) !}{\prod_{j=1}^{\ell} \prod_{i=1}^{n} k_{i j} !} \frac{1}{\ell !} \prod_{j=1}^{\ell} \mathscr{F}\left(x_{1}^{k_{i j}}, \ldots, x_{n}^{k_{n j}}\right)
$$

as formal power series in $\lambda$. The inner summation is over all irreducible $n \times \ell$ matrices $k^{(\ell)}$ whose entries are nonnegative integers.

Proof. Write

$$
Z(\Lambda \mid \psi)=\int d \mu_{\vartheta}(\phi) \prod_{x \in \Lambda} e^{-\lambda \mathscr{V}\left(\varphi_{x}\right)}
$$

where $\mathscr{V}$ depends on $\psi$, and $\varphi_{x}=a^{d} \varphi(x), d=\frac{1}{2}(v-2)$. Both the Mayer amplitude and the Feynman amplitude may be expressed as truncated expectation values

$$
\mathscr{M}\left(x_{1}, \ldots, x_{n}\right)=\frac{a^{-v n}}{n !}\left[\left\langle\prod_{i=1}^{n}\left[e^{-\lambda \mathscr{V}\left(\varphi_{x_{i}}\right)} ;\right]\right\rangle_{v}-\delta_{1, n}\right]
$$

and

$$
\mathscr{F}\left(y_{1}, \ldots, y_{k}\right)=\frac{1}{k !}\left\langle\prod_{j=1}^{k}\left[-\lambda \mathscr{V}\left(\varphi_{y_{j}}\right) ;\right]\right\rangle_{v} .
$$

In the first expression one expands

$$
e^{-\lambda \mathscr{V}\left(\varphi_{x}\right)}=\sum_{\ell=0}^{\infty} \frac{1}{\ell !}\left[-\lambda \mathscr{V}\left(\varphi_{x}\right)\right]^{\ell}
$$

Then one expresses the resulting "partially truncated" expectation value of a product of factors $-\lambda \mathscr{V}(\varphi$.) in terms of completely truncated expectation values with the help of the following

\section{Lemma 4.2.}

$$
\begin{aligned}
& \left\langle\prod_{i=1}^{n}\left[F_{i}\left(\varphi_{y_{i}}\right)^{n_{i}} ;\right]\right\rangle_{v} \\
& \quad=\sum_{\ell \geqq 1} \sum_{\substack{k^{(\ell)} \\
\sum_{j=1}^{\ell} k_{i j}^{(\ell)}=n_{i}}} \frac{\prod_{i=1}^{n} n_{i} !}{\prod_{i=1}^{n} \prod_{j=1}^{\ell} k_{i j}^{(\ell) !}} \frac{1}{\ell !} \prod_{j=1}^{\ell}\left\langle\prod_{i=1}^{n}\left[F_{i}\left(\varphi_{y_{i}}\right) ;\right]^{k_{i j}^{(\ell)}}\right\rangle_{v} .
\end{aligned}
$$

Summation is over irreducible $n \times \ell$ matrices $k^{(\ell)}$ as above which satisfy indicated constraint.

Sketch of the Proof of Lemma 4.2. One considers first the ordinary expectation value

$$
\left\langle F_{1}\left(\varphi_{y_{1}}\right)^{n_{1}} \ldots F_{n}\left(\varphi_{y_{k}}\right)^{n_{k}}\right\rangle .
$$

Using the defining relation (2.18) of truncated expectation values, this can be expressed as a truncated expectation value of $k$ factors $F_{i}\left(\varphi_{y_{i}}\right)^{n_{i}}$ and also as a truncated expectation value of $N=\sum n_{i}$ factors $F_{i}\left(\varphi_{y_{i}}\right)$. It suffices to show that the two expressions become equal when Eq. (4.14) is inserted into the first. This is so 
because of uniqueness of the activities of a polymer system, cf. remark after Eq. (2.18). After some combinatorics it is found that they are indeed equal. Details are spelled out in [21].

We mentioned in the introduction that standard Feynman perturbation theory may be put on a staggered lattice, graph by graph. The new graphs will involve nonlocal vertices which can connect different layers, and propagators that link only points in the same layer. Using this, the results of this section could be extended to the Mayer amplitudes on a staggered lattice. We think, however, that they are good enough as they stand to give the general idea.

To make sure that there is no misunderstanding, let us emphasize one fact at the end of this perturbation theoretical section. Mayer amplitudes are not defined as divergent sums of Feynman amplitudes. They are defined as perfectly meaningful truncated expectation values like (4.13). These well defined expressions admit an asymptotic power series expansion in $\lambda$ whose coefficients are Feynman amplitudes.

\section{The Special Case of a Lattice with only One Point}

The partition function for $\lambda \phi^{4}$-theory on a lattice $\Lambda=\{0\}$ that consists of a single point is given by a convergent 1-dimensional integral

$$
Z(\{0\} \mid \psi)=(2 \pi v)^{-1 / 2} \int_{\mathbb{R}} d \varphi \exp \left[\begin{array}{c}
\left.-\frac{1}{2} v^{-1} \varphi^{2}-\frac{\lambda}{4 !}(\varphi+\psi)^{4}+\frac{1}{2} \delta m^{2}(\varphi+\psi)^{2}+\delta e\right], \\
\lambda>0, v>0, \text { real }
\end{array}\right.
$$

Its perturbation expansion in powers of $\lambda$ will be divergent no matter how $\delta m^{2}$ and $\delta e$ depend on $\lambda$, because the integral makes no sense for $\lambda<0$, and is therefore not analytic at $\lambda=0[23]$.

On the other hand, a Mayer expansion for this model amounts to "do nothing". There is only a single Mayer amplitude, which is given by a convergent 1-dimensional integral, and

$$
Z(\{0\} \mid \psi)=\mathscr{M}(0 \mid \psi) \exp \left[-\frac{\lambda}{4 !} \psi^{4}\right] .
$$

Greens functions are obtained by differentiation at $\psi=0$. They do not involve divergent sums.

\section{Appendix A. \\ Generating Function for Free-Propagator-Amputated Greens Functions}

In a theory with free propagator $v$, the connected, free-propagator-amputated Greens functions $G_{c}\left(\underline{z}_{1}, \ldots, \underline{z}_{n}\right)$ are related to the corresponding unamputated Greens function $G_{c}\left(z_{1}, \ldots, z_{n}\right)$ by

and

$$
\begin{aligned}
& G_{c}\left(z_{1}, \ldots, z_{n}\right)=\int_{z_{1}^{\prime}} \ldots \int_{z_{n}^{\prime}} v\left(z_{1}, z_{1}^{\prime}\right) \ldots v\left(z_{n}, z_{n}^{\prime}\right) G_{c}\left(\underline{z}_{1}^{\prime}, \ldots, \underline{z}_{n}^{\prime}\right) \quad(n \geqq 3) \\
& G_{c}\left(z_{1}, z_{2}\right)=v\left(z_{1}, z_{2}\right)+\int_{z_{1}^{\prime} z_{2}^{\prime}} v\left(z_{1}, z_{1}^{\prime}\right) v\left(z_{2}, z_{2}^{\prime}\right) G_{c}\left(\underline{z}_{1}^{\prime}, \underline{z}_{2}^{\prime}\right)=G\left(z_{1}, z_{2}\right) .
\end{aligned}
$$


Amputation is indicated by underlining the argument. The Greens functions can be obtained by differentiation from generating functions

$$
\begin{aligned}
G_{c}\left(z_{1}, \ldots, z_{n}\right) & =\left.\frac{\delta^{n}}{\delta J\left(z_{1}\right) \ldots \delta J\left(z_{n}\right)} G(J)\right|_{J=0}, \\
G_{c}\left(\underline{z}_{1}, \ldots, \underline{z}_{n}\right) & =\left.\frac{\delta^{n}}{\delta \psi\left(z_{1}\right) \ldots \delta \psi\left(z_{n}\right)} F(\psi)\right|_{\psi=0} .
\end{aligned}
$$

The above relations correspond to the following relation between generating functions

$$
F(\psi=v J)=G(J)-\frac{1}{2} J v J
$$

in obvious notation. In a theory with interaction Lagrangian $V$, the generating function $G(J)$ is the logarithm of the partition function in the presence of a source

$$
e^{G(J)}=\int d \mu_{v}(\phi) e^{-V(\phi)+J \phi} .
$$

The free field measure is

$$
d \mu_{\vartheta}(\phi)=(\operatorname{det} 2 \pi v)^{-1 / 2} e^{-\frac{1}{2} \phi v^{-1} \phi} \mathscr{D} \phi .
$$

It follows that

$$
\begin{aligned}
d \mu_{\vartheta}(\phi-\psi) & =e^{-\frac{1}{2} \psi v^{-1} \psi+\phi v^{-1} \psi} d \mu_{\vartheta}(\phi) \\
& =e^{-\frac{1}{2} J v J+\phi J} d \mu_{v}(\phi) \text { if } \quad \psi=v J
\end{aligned}
$$

Let us define

$$
Z(\psi)=\int d \mu_{v}(\phi) e^{-V(\phi+\psi)}
$$

We show that

$$
F(\psi)=\ln Z(\psi)
$$

obeys the relation (A.4).

Making a shift of the field in (A.8) and using Eq. (A.7) we obtain

$$
\begin{aligned}
Z(\psi) & =\int d \mu_{v}(\phi) e^{-\frac{1}{2} J v J+\phi J} e^{-V(\phi)} \\
& =e^{G(J)-\frac{1}{2} J v J} \quad \text { if } \quad \psi=v J
\end{aligned}
$$

Making a shift in the field, we must verify that boundary conditions at infinity are preserved. They are preserved if $\psi$ falls to zero at infinity. This will be the case for locally supported source $J$ if $v$ is a massive propagator. Equation (A.10) agrees with the desired relation (A.4). q.e.d.

The connected free-propagator-amputated Greens functions are not 1-particle irreducible, and must not be confused with the so-called vertex-functions $\equiv$ fullpropagator-amputated 1-particle irreducible Greens functions whose generating function is related to $G(J)$ by a Legendre transform. 


\section{Appendix B. Block Spins, Propagators, and Kernels $\mathscr{A}$}

We will follow the procedure of Kupiainen and Gawedzki [9] to carry out the split (1.5), (1.6) of the field $\phi$ and propagator $v>0$ on base space. No summation will be implied by repeated indices.

Let us assume that there is a finest lattice $\Lambda_{N}=$ base space. Results for continuum base space may be obtained by letting $N \rightarrow \infty$. As we have mentioned in the main text, the points $x \in \Lambda_{j}$ may be considered as blocks of side length $a_{j}$ in base space.

Given a field $\phi=\phi_{N}$ on base space, we define the blockspin $\Phi^{j}(x)$ on $\Lambda_{j}$ as block average of $\phi$

$$
\Phi^{j}(x)=\underset{z \in x}{a v} \phi(z) \equiv C^{j} \phi(x)
$$

Accordingly, the free propagators [covariances] $v$ and $u_{j}$ of the [Gaussian] fields $\phi$ and $\Phi^{j}$ are related by

$$
u_{j}=C^{j}{ }^{j *}>0 \text {. }
$$

* is the adjoint with respect to the scalar products $($,$) that are furnished by$ integration over base space and $\Lambda_{j}$, respectively. The field $\phi$ may be split into a part that is determined by the block spin $\Phi^{j}$, and a fluctuation field $\zeta^{j+1}$ on base space that has zero block average.

$$
\phi=\mathscr{A}^{j} \Phi^{j}+\zeta^{j+1} \quad \text { with } \quad C^{j \zeta^{j+1}}=0 .
$$

In the special case $j=N$ this holds with $\zeta^{N+1}=0, \mathscr{A}^{N}=1$. The kernel $\mathscr{A}^{j}$ is chosen as

$$
\mathscr{A}^{j}=v C^{j *} u_{j}^{-1}
$$

so that the two pieces on the right-hand side are orthogonal with respect to the scalar product $\langle$,$\rangle that is specified by the inverse propagator$

$$
\langle f, g\rangle=\left(f, v^{-1} g\right) \text {. }
$$

Evidently

$$
C^{j} \mathscr{A}^{j}=1
$$

We will define the pieces $\varphi^{k}$ of the field $\phi$ in such a way that

$$
\zeta^{j}=\sum_{k \geqq j} \mathscr{A}^{k} \varphi^{k}
$$

Expressed in words, the fluctuation field $\zeta^{j}$ in the sum of the parts of the field $\varphi$ with "frequency" $k \geqq j$ (appropriately transported to base space with kernels $\mathscr{A}^{k}$ ). For $j=N+1$ this relation holds with $\varphi^{N+1}=0$. Insert (B.6) into (B.3) to obtain

$$
\phi=\mathscr{A}^{j} \Phi^{j}+\sum_{k>j} \mathscr{A}^{k} \varphi^{k}
$$

Suppose this relation holds for some $j$. We determine $\varphi^{j}$ so that it holds for $j-1$.

To this end we define the operation $C_{j-1, j}$ which averages functions on $\Lambda_{j}$ over cells of $\Lambda_{j-1}$. Evidently, definition (B.1) implies

$$
\Phi^{j-1}=C_{j-1, j} \Phi^{j}
$$


We split the field $\Phi^{j}$ into a piece that is determined by its block average $\Phi^{j-1}$ and a part $\varphi^{j}$ on $\Lambda_{j}$ whose block average vanishes,

$$
\Phi^{j}=\mathscr{A}_{j, j-1} \Phi^{j-1}+\varphi^{j}
$$

with

$$
C_{j-1, j} \varphi^{j}=0 .
$$

The kernel $\mathscr{A}_{j, j-1}$ is chosen as

$$
\mathscr{A}_{j, j-1}=u_{j} C_{j-1, j}^{*} u_{j-1}^{-1},
$$

so that

$$
\mathscr{A}^{j} \mathscr{A}_{j, j-1}=\mathscr{A}^{j-1} .
$$

Inserting Eqs. (B.9) and (B.12) into (B.7), we see that (B.7) remains true for $j-1$, as promised.

Equation (B.9), together with definitions (B.1) of the block spins and (B.11), (B.2) of the kernel $\mathscr{A}$.., defines the pieces $\varphi^{j}$ of the field for all $j \geqq 1$ in such a way that (B.7) holds for all $j$. For $j=0$ this equation is of the form (1.5) if we set, finally

$$
\varphi^{0}=\Phi^{0} .
$$

$\mathscr{A}(z, x)$ is the integral kernel of $\mathscr{A}^{j}$ if $x \in \Lambda_{j}$, viz.

$$
\mathscr{A}^{j} f(z)=\int_{x \in \Lambda_{j}} \mathscr{A}(z, x) f(x) \text {. }
$$

It remains to determine the free propagator [covariance] $v$ of $\varphi^{j}$. Regard $\varphi^{j}$ as a function of $\phi$. We may then use the definition (B.9), (B.13) of $\varphi^{j}$ to compute

$$
v(x, y)=\left\langle\varphi^{j}(x) \varphi^{k}(y)\right\rangle \text { for } x \in \Lambda_{j}, y \in \Lambda_{k} .
$$

Using the relation (B.8) and the known propagator $u_{j}$ of $\Phi^{j}$, it turns out to be of the form

$$
v(x, y)=\delta_{j k} v^{j}(x, y)
$$

with

$$
\begin{aligned}
v^{j} & =u_{j}-u_{j} C_{j-1, j}^{*} u_{j-1}^{-1} C_{j-1, j} u_{j} \text { for } j \geqq 1, \\
v^{0} & =u_{0} .
\end{aligned}
$$

$v$ is positive semidefinite by definition (B.15). It follows from Eq. (B.15) and (1.15), viz.

$$
\phi=\sum_{j=0}^{N} \mathscr{A}^{j} \varphi^{j}
$$

that the relation (1.6) between propagators holds. That is

$$
v=\sum_{j} \mathscr{A}^{j} v^{j} \mathscr{A}^{j *} \equiv \mathscr{A} v \mathscr{A}^{*} .
$$

Decay properties of kernels $\mathscr{A}$ and propagators $v$ were studied in [9, 22]. Finally we should transcribe Gaussian measures. We claim that

$$
\int d \mu_{v}(\phi) F(\phi)=\int d \mu_{v}(\varphi) F(\mathscr{A} \varphi) .
$$


It suffices to verify this for $F(\phi)=e^{i(q, \phi)}$. Using the known Fourier transformation (3.16) of Gaussian measures, one finds

$$
\int d \mu_{v}(\phi) e^{i(q, \phi)}=e^{-\frac{1}{2}(q, v q)}=e^{-\frac{1}{2}\left(q, \mathscr{A}^{v} \mathscr{A}^{*} q\right)},
$$

while

$$
\int d \mu_{v}(\varphi) e^{i(q, \mathscr{A} \varphi)}=e^{-\frac{1}{2}(\mathscr{A} *, v \mathscr{A} *)}=e^{-\frac{1}{2}\left(q, \mathscr{A} v \mathscr{A}_{q}^{*}\right)} \cdot \quad \text { q.e.d. }
$$

Using Eq. (B.19) it follows from the formula (A.8) for the generating function $\ln Z(\psi)$ of connected Greens functions, and definitions (1.7), (1.9a) that the relation (1.10) for $\ln Z(\psi)$ holds for finite volume and cutoff.

\section{Appendix C. Proof of the Recursion Relation (Proposition 3.1)}

We start from the Kirkwood-Salsburg Eq. (3.18) which defines the generalized molecular activities $B^{Y}$. Iterating it once we obtain, for $w \notin X+Y$

with

$$
\mathscr{Z}^{Y}\left(X+w \mid \psi+\mathscr{A} \varphi_{X+w}\right)=\sum_{\substack{P \in P \subseteq X\\}} C^{Y}\left(P+w \mid \psi, \varphi_{P+w}\right) \mathscr{Z}^{Y}\left(X-P \mid \psi+\mathscr{A} \varphi_{X-P}\right)
$$

$$
\begin{aligned}
C^{Y}\left(P+w \mid \psi, \varphi_{P+w}\right)= & B^{Y}\left(P+w \mid \psi, \varphi_{P+w}\right) \\
& +\sum_{\substack{Q \\
x \in \mathcal{Q} \cong P}} B^{Y}\left(Q \mid \psi, \varphi_{Q}\right) B^{Y}\left(P-Q+w \mid \psi, \varphi_{P-Q+w}\right)
\end{aligned}
$$

$C^{Y}(\ldots)$ can be determined recursively from Eq. (C.1). Therefore, two expressions for $C^{Y}(\ldots)$ which both satisfy Eq. (C.1) must necessarily be equal. By definition (3.17) we have

$$
\mathscr{Z}^{Y}\left(X+w \mid \psi+\mathscr{A} \varphi_{X+w}\right)=\mathscr{Z}^{Y+w}\left(X \mid \psi^{\prime}+\mathscr{A} \varphi_{X}\right), \quad \psi^{\prime}=\psi+\mathscr{A} \varphi_{w} .
$$

Inserting the Kirkwood-Salsburg Eq. (3.18) for the right-hand side gives

But

$$
\mathscr{Z}^{Y}\left(X+w \mid \psi+\mathscr{A} \varphi_{X+w}\right)=\sum_{\substack{P \\ x \in P \cong X}} B^{Y+w}\left(P \mid \psi^{\prime}, \varphi_{P}\right) \mathscr{Z}^{Y+w}\left(X-P \mid \psi^{\prime}+\mathscr{A} \varphi_{X-P}\right) .
$$

$$
\begin{aligned}
\mathscr{Z}^{Y+w}\left(X-P \mid \psi^{\prime}+\mathscr{A} \varphi_{X-P}\right)=\mathscr{Z}^{Y}\left(X-P+w \mid \psi+\mathscr{A} \varphi_{X-P+w}\right) \\
=\sum_{w \in Q \subseteq \sum_{X-P+w}} B^{Y}\left(Q \mid \psi, \varphi_{Q}\right) \mathscr{Z}^{Y}\left(X-P+w-Q \mid \psi+\mathscr{A} \varphi_{X-P+w-Q}\right)
\end{aligned}
$$

by the Kirkwood-Salsburg equation. Inserting this and setting

$$
P+Q-w=R \quad \text { so that } \quad x \in R \subseteq X, Q=R-P+w, P \subseteq R,
$$

we obtain

$$
\begin{aligned}
\mathscr{Z}^{Y}\left(X+w \mid \psi+\mathscr{A} \varphi_{X+w}\right)= & \sum_{\substack{R \\
x \in R \cong X}}\left\{\sum_{\substack{P \\
x \in P \cong R}} B^{Y+w}\left(P \mid \psi^{\prime}, \varphi_{P}\right) B^{Y}\left(R-P+w \mid \psi, \varphi_{R-P+w}\right)\right\} \\
& \cdot \mathscr{Z}^{Y}\left(X-R \mid \psi+\mathscr{A} \varphi_{X-R}\right) .
\end{aligned}
$$

This is another equation of the form (C.2). Equating the coefficient in \{\} to $C^{Y}(R+w \mid \ldots)$ produces the recursion relation (Proposition 3.1). 
Acknowledgements. This work was begun while one of the authors (G.M.) was at the Institute for Advanced Studies in Princeton, NJ. He would like to thank S. Adler and R. Dashen for their hospitality, and the Deutsche Forschungsgemeinschaft for a travel grant. He would also like to thank P.K. Mitter for the invitation to a topical meeting on renormalization group problems in Paris in spring 1984, and its participants for many stimulating discussions. Finally we would like to thank G. Gallavotti and F. Nicoló for providing a copy of their paper [12] before publication.

\section{References}

1. Symanzik, K.: Euclidean quantum field theory. I. Equations for a scalar model. J. Math. Phys. 7, $510(1966)$

2. Brydges, D.: A rigorous approach to Debye screening in dilute classical Coulomb systems. Commun. Math. Phys. 58, 313 (1978)

Brydges, D., Federbush, P.: Debye screening. Commun. Math. Phys. 73, 197 (1980)

3. Göpfert, M., Mack, G.: Iterated Mayer expansions for classical gases at low temperatures. Commun. Math. Phys. 81, 97 (1981)

4. Göpfert, M., Mack, G.: Proof of confinement of static quarks in 3-dimensional U(1) lattice gauge theory for all values of the coupling constant. Commun. Math. Phys. 82, 545 (1982)

5. Glimm, J., Jaffe, A.: Positivity of the $\varphi_{3}^{4}$-Hamiltonian. Fortschr. Phys. 21, 327 (1973)

6. Magnen, J., Seneor, R.: The infrared behavior of $(\nabla \phi)_{3}^{4}$ theory. Ann. Phys. 152, 130 (1984). See also: Phase space cell expansion and Borel summability of the Euclidean $\varphi_{3}^{4}$-theory. Commun. Math. Phys. 56, 237 (1977)

7. Battle, G., Federbush, P.: A phase cell cluster expansion for Euclidean field theories. Ann. Phys. 142, 95 (1982); A phase cell cluster expansion for a hierarchial $\phi_{3}^{4}$-model. Commun. Math. Phys. 88, 263 (1983)

8. Battle, G.: A new combinatoric estimate for cluster expansions. Commun. Math. Phys. 94, 133 (1984)

Battle, G., Federbush, P.: A note on cluster expansions, tree graph identities, extra $1 / N$ ! factors!!! Lett. Math. Phys. 8, 55-57 (1984)

9. Kupiainen, A., Gawedzki, K.: A rigorous block spin approach to massless lattice theories. Commun. Math. Phys. 77, 31 (1980); Block spin renormalization group for dipole gas and $(\nabla \phi)^{4}$. Ann. Phys. 147, 198 (1983)

10. Feldman, J., Magnen, J., Rivasseau, V., Seneor, R.: Methodes pour la theorie constructive des champs renormalisables asymptotiquement libres, preprint école polytechnique, Palaiseau, June 1984

11. Kupiainen, A., Gawedzki, K.: Massless lattice $\lambda \phi^{4}$-theory: A nonperturbative control of a renormalizable model. Preprint IHES Bures-sur-Yvette, June 1984

12. Gallavotti, G., Nicoló, F.: Renormalization theory in four dimensional scalar fields. Rutgers preprint, spring 1984

13. Bogolubov, N.N., Shirkov, D.V.: Introduction to the theory of quantized fields. New York: Interscience 1959

14. Zimmermann, W.: Convergence of Bogolubov's method of renormalization in momentum space. Commun. Math. Phys. 15, 208 (1969)

15. Wilson, K.: Renormalization group and strong interactions. Phys. Rev. D 3, 1818 (1971)

16. Gruber, C., Kunz, H.: General properties of polymer systems. Commun. Math. Phys. 22, 133 (1971). See also: Gallavotti, G., Miracle Solé, S.: Correlation functions of a lattice system. Commun. Math. Phys. 7, 274 (1968)

17. Ruelle, D.: Statistical mechanics. New York: Benjamin 1969

18. Seiler, E.: Gauge theories as a problem in constructive quantum field theory and statistical mechanics. Lecture Notes in Physics, Vol. 159. Berlin, Heidelberg, New York: Springer 1982 Fredenhagen, K., Marcu, M.: Charged states in $\mathbb{Z}_{2}$ gauge theory. Commun. Math. Phys. 92,81 (1983) (Appendix)

Göpfert, M.: Das Peierls Argument für Z(2)-Gitter-Eichtheorien. Diplomarbeit Hamburg 1979 (unpublished) 
Malyshev, V.A.: Uniform cluster expansions for lattice models. Commun. Math. Phys. 64, 131 (1979);

Münster, G.: High temperature expansion for the free energy of vortices and the string tension in lattice gauge theory. Nucl. Phys. B 180 [FS2], 23 (1981)

19. Glimm, J., Jaffe, A.: Quantum physics. Berlin, Heidelberg, New York: Springer 1981

20. Brydges, D., Federbush, P.: A new form of the Mayer expansion in classical statistical mechanics. J. Math. Phys. 19, 2604 (1978)

21. Pordt, A.: Mayer-Entwicklungen für Euklidische Gitterfeldtheorie: Konvergenzeigenschaften und Beziehung zur Störungstheorie. Diplomarbeit Hamburg 1984

22. Balaban, T.: Regularity and decay of lattice Green's functions. Commun. Math. Phys. 89, 571 (1983)

23. Dyson, F.: Divergence of perturbation theory in quantum electrodynamics. Phys. Rev. 85, 631 (1952)

Communicated by A. Jaffe

Received August 31, 1984; in revised form September 21, 1984 